\title{
Identification of Specific Oral and Gut Pathogens in Full Thickness Colon of Colitis Patients: Implications for Colon Motility
}

OPEN ACCESS

Edited by:

Nurul-Syakima Ab Mutalib,

UKM Medical Molecular Biology Institute (UMBI), Malaysia

Reviewed by:

Christopher L. Hemme, University of Rhode Island,

United States

Chenyang Wang,

Nanjing University Medical School,

China

${ }^{*}$ Correspondence:

Pandu R. Gangula

pgangula@mmc.edu

†These authors have contributed equally to this work

Specialty section: This article was submitted to

Microbial Symbioses,

a section of the journal

Frontiers in Microbiology

Received: 11 September 2018 Accepted: 11 December 2018

Published: 04 January 2019

Citation: Dinakaran V, Mandape SN, Shuba K, Pratap S, Sakhare SS, Tabatabai MA,

Smoot DT, Farmer-Dixon CM,

Kesavalu LN, Adunyah SE,

Southerland JH and Gangula PR (2019) Identification of Specific Oral and Gut Pathogens in Full Thickness Colon of Colitis Patients: Implications

for Colon Motility.

Front. Microbiol. 9:3220.

doi: 10.3389/fmicb.2018.03220

\begin{abstract}
Vasudevan Dinakaran ${ }^{1+}$, Sammed N. Mandape ${ }^{2 \dagger}$, Kristina Shuba ${ }^{1}$, Siddharth Pratap ${ }^{2}$, Shruti S. Sakhare ${ }^{2}$, Mohammad Ali Tabatabai ${ }^{3}$, Duane T. Smoot ${ }^{4}$, Cherae M. Farmer-Dixon ${ }^{1}$, Lakshmyya N. Kesavalu ${ }^{5}$, Samuel Evans Adunyah ${ }^{6}$, Janet Hayes Southerland ${ }^{7}$ and Pandu R. Gangula ${ }^{1 *}$

${ }^{1}$ Department of ODS \& Research, School of Dentistry, Meharry Medical College, Nashville, TN, United States, ${ }^{2}$ Bioinformatics Core, School of Graduate Studies/Research \& School of Medicine, Meharry Medical College, Nashville, TN, United States, ${ }^{3}$ Department of Public Health, School of Graduate Studies \& Research, Meharry Medical College, Nashville, TN, United States, ${ }^{4}$ Department of Internal Medicine, Division of Gastroenterology \& Hepatology, Meharry Medical College, Nashville, TN, United States, ${ }^{5}$ Department of Periodontology, College of Dentistry, University of Florida, Gainesville, FL, United States, ${ }^{6}$ Department of Biochemistry, Cancer Biology, Neuroscience \& Pharmacology, Meharry Medical College, Nashville, TN, United States, ${ }^{7}$ Department of Nutrition Metabolism \& Oral Surgery, University of Texas Medical Branch at Galveston, Galveston, TX, United States
\end{abstract}

Impaired colon motility is one of the leading problems associated with inflammatory bowel disease (IBD). An expanding body of evidence supports the role of microbiome in normal gut function and in progression of IBD. The objective of this work is to determine whether diseased full thickness colon specimens, including the neuromuscular region (critical for colon motility function), contain specific oral and gut pathogens. In addition, we compared the differences in colon microbiome between Caucasians (CA) and African Americans (AA). Thirty-nine human full thickness colon (diseased colon and adjacent healthy colon) specimens were collected from Crohn's Colitis (CC) or Ulcerative Colitis (UC) patients while they underwent elective colon surgeries. We isolated and analyzed bacterial ribosomal RNA ( $\mathrm{RRNA}$ ) from colon specimens by amplicon sequencing of the $16 S$ rRNA gene region. The microbiome proportions were quantified into Operational Taxonomic Units (OTUs) by analysis with Quantitative Insights Into Microbial ecology (QIIME) platform. Two hundred twenty-eight different bacterial species were identified by QIIME analysis. However, we could only decipher the species name of fifty-three bacteria. Our results show that proportion of non-detrimental bacteria in CC or UC colon samples were altered compared to adjacent healthy colon specimens. We further show, for the first time in full thickness colon specimens, that microbiome of CC and UC diseased specimens is dominated by putative oral pathogens belonging to the Phyla Firmicutes (Streptococcus, Staphylococcus, Peptostreptococcus), and Fusobacteria (Fusobacterium). In addition, we have identified patterns of differences in microbiome levels between CA and AA specimens with potential implications for health disparities research. Overall, our results suggest a significant association between oral and gut microbes in the modulation of colon motility in colitis patients.

Keywords: colitis, colon motility, nitric oxide (NO), antioxidants, oral microbiome, operational taxonomic units (OTUs), gut microbiome 


\section{INTRODUCTION}

Inflammatory bowel disease (IBD) is comprised of Crohn's disease / Crohn's colitis (CC) and Ulcerative colitis (UC). The term Colitis, refers to general inflammation of the inner lining of the colon arising from numerous underlying causes including idiopathic infection, IBD (either CC or UC), ischemic colitis, allergic reactions, and/or microscopic colitis. Distally, gingivitis, and periodontal disease are chronic inflammatory gum diseases associated with orange, red, yellow, purple, and green complex bacterial infections in sub-gingival areas of oral cavity (Popova et al., 2013).

Previous studies have shown that periodontal disease (PD) is a significant risk factor and contributor to many systemic diseases, including IBD (Vavricka et al., 2013). Several factors including genetic, dietary, and environmental factors could influence the pathogenesis of microbiome (oral and gut) which in turn may increase the incidence of periodontitis and IBD (Lira-Junior and Figueredo, 2016; Agossa et al., 2017). In addition, Porphyromonas gingivalis known to cause PD altered the gut microbiota leading to increased gut epithelial permeability and endotoxemia, which causes systemic inflammation (Hajishengallis, 2015). In addition, many earlier studies have shown intestinal colonization of oral bacteria in the pathogenesis of IBD (Strauss et al., 2011; Atarashi et al., 2017).

Innumerable number of studies have shown that the gut microbiome including Phyla Proteobacteria, Firmicutes, and Bacteroidetes contribute to normal gut function (Mariat et al., 2009; Koliada et al., 2017; Walker et al., 2018; Zhao et al., 2018). Colon motility is mainly regulated by neuromuscular portion of the colon and this was shown to be impaired in colitis patients; putatively due to a reduction in neuronal nitric oxide (NO) synthase (nNOS) protein expression and/or neuronal degeneration (Bassotti et al., 2014; Gangula et al., 2017). Previous studies have analyzed the microbiome in feces and/or colon mucosal biopsy specimens of colitis patients (Gibson et al., 1991; Bibiloni et al., 2006). However, the relationship/interaction between oral and gut bacteria in the development and/or exacerbation of inflammatory disease in the colon (containing neuromuscular tissue) was under studied. In addition, data is limited on how oral bacteria interact with and influence the large intestinal flora, thereby contributing to colitis. Since motility of the colon is impaired in colitis patients and neuromuscular tissue play a role in the motility function (Geboes and Collins, 1998; Poli et al., 2001), we hypothesize that the interaction between oral and gut microbiome may play a significant role in the inflammatory processes associated with the development and progression of colitis seen in certain patient populations. Furthermore, we hypothesize that difference in microbiome may exist between CA and AA colitis patients, potentially contributing to health disparities in IBD.

\section{METHODS}

\section{Ethics Statement}

The participants provided both written and verbal informed consent to Collaborative Human Tissue Networking (CHTN)
Consortium to collect specimens while they underwent elective colon surgeries.

\section{Collection of Specimens}

Frozen full thickness colon specimens were obtained from Cooperative Human Tissue Networking (CHTN). Thirty-nine human full thickness colon (moderate to severe diseased colon and adjacent healthy colon) specimens were collected from CC and UC male and female patients (ages between 18 and 75 years old) while they underwent elective colon surgeries. The specimens include Ulcerative $(n=13)$, Crohn's $(n=13)$ and adjacent healthy $(n=13)$ specimens. Characteristics of participants included Caucasians (CA) $(n=30)$ and African Americans (AA) $(n=9)$. CC male and female patients presented with symptoms like fever, fatigue, diarrhea, blood in stool, mouth sores, abdominal cramping, and pain around the anus, reduced appetite, and weight loss. While UC male and female patients presented with additional signs like rectal pain, rectal bleeding, and inability to defecate despite urgency.

\section{Extraction of DNA, Amplification of 16S rRNA Gene and Amplicon Sequencing}

DNA extraction and microbial analysis were performed in the University of North Carolina at Chapel Hill School of Medicine Microbiome Core Facility (UNC: MC). We identified a conserved region of the 16S rRNA gene of 550 bp to amplify. This encompassed variable regions V3-V4 from the colon genomic DNA using primers $16 \mathrm{~S}$ rRNAF 5'-AGAGTTTGATCCTGGCTCAG-3' and 16S rRNA-R $5^{\prime}$-GCTGCCTCCCGTAGGAGT-3' and overhang adapter sequences appended to the primer pair for compatibility with Illumina index and sequencing adapters. Briefly, each 16SrRNA amplicon was purified using AMPure XP reagent (Beckman Coulter, Indianapolis, IN, USA). Specifically, each sample was amplified using a limited cycle PCR program, adding Illumina sequencing adapters and optional dual-index barcodes [index 1(i7) and index 2(i5)] (Illumina, San Diego, CA, USA) to the amplicon target. The final libraries were purified using AMPure $\mathrm{XP}$ reagent, quantified and normalized prior to pooling. The DNA library pool was denatured with $\mathrm{NaOH}$, diluted with hybridization buffer and heat denatured before loading on to the MiSeq reagent cartridge and to the MiSeq instrument (Illumina). The standard Illumina paired-end 250 base pair (PE250) protocol was used for sequencing the16S rRNA amplicons (Illumina, CA, USA).

\section{Processing of Sequence Reads}

Data was analyzed and microbial proportions using Operational Taxonomic Units (OTUs) were determined using Quantitative Insights Into Microbial ecology (QIIME) pipeline (Caporaso et al., 2010a) in the Meharry Medical College Bioinformatics Core. Briefly, generated raw reads were preprocessed for adapter removal. Processed sequence reads were obtained as fastq files and were converted into fasta, quality and flow files using Mothur package (Schloss et al., 2009). The initial number of fasta sequences obtained were $31,09,793$. First, the fasta files were cleaned of host reads by mapping on to $9 \mathrm{~mm}$ mouse 
genome. Then, the primer sequences and barcode sequences were removed, demultiplexed and quality filtered. The number of high quality sequences remaining after quality filtering was $16,64,769$. The OTUs were picked by de novo strategy. The high quality sequences were clustered at $97 \%$ identity using UCLUST inbuilt in QIIME pipeline to generate 3994 OTUs and taxonomy was assigned to OTU representative sequences using UCLUST (Edgar, 2010). The picked sequences were aligned using PyNAST aligner (Caporaso et al., 2010b). The chimeric sequences and singleton OTUs were removed using ChimeraSlayer (Haas et al., 2011). We constructed a phylogenetic tree for the sequences using FastTree version 2.1.3 (data not shown) (Price et al., 2010). Next, an OTU table was constructed and taxa were summarized using the 894 OTUs obtained from QIIME pipeline. $\alpha$ diversity metrics was computed using Chaol (abundance-based richness estimator) and Shannon analysis (diversity index) and Rarefaction plots were constructed (data not shown). $\beta$-diversity metrics was computed using weighted and unweighted Principal Coordinates Analysis (PCoA) (data not shown) (Gower, 2005). A Taxonomic Summary Bar plot showing OTUs assigned to Phyla-level taxonomy per sample was subsequently constructed (Figure 1). Bar Plots showing the relative abundance of bacteria at the Phyla-level between races, diseased tissue and healthy tissue groups is shown in Figure 3. Sample-specific sequences were deposited in the MGRAST database (accession number: b3b851ba2c6d676d343739393937332e33) and was assigned an MG-RAST project ID (mgs675214) (Keegan et al., 2016). In addition, sample-specific sequences were deposited in the NCBI (BioProject: PRJNA496071).

The pathogenic and beneficial oral and gut bacteria were identified using the NCBI Genome database (https://www.ncbi. nlm.nih.gov/genome/). This analysis was performed to assess the pathogenic and healthy bacterial proportions in human full thickness colon specimens (Tables 1-3).

\section{Statistical Analysis and Evaluation}

Statistical analysis was performed between the healthy and diseased groups and based on race classification ( $n=13 \mathrm{CC}$, $n=13 \mathrm{UC}, n=13$ non-disease healthy patients, $n=30 \mathrm{CA}$ and $n=9 \mathrm{AA}$ ). A non-parametric Mann-Whitney $U$ Test $p$ value $<0.05$ of bacterial $16 \mathrm{~S}$ rRNA OTUs between the groups was considered statistically significant. IBM SPSS software package version 23 (IBM Analytics, USA) was used to conduct statistical analysis.

\section{RESULTS}

\section{Relative Abundance Analysis}

QIIME analysis showed about two hundred twenty-eight bacterial species in entire 39 specimens (Tables 1-5). However, non-ambiguous annotation at the species name resulted in fifty-three bacterial identifications. The dominant phyla across all samples (both diseased and healthy specimens) were Bacteroidetes (46.92\%), followed by Firmicutes

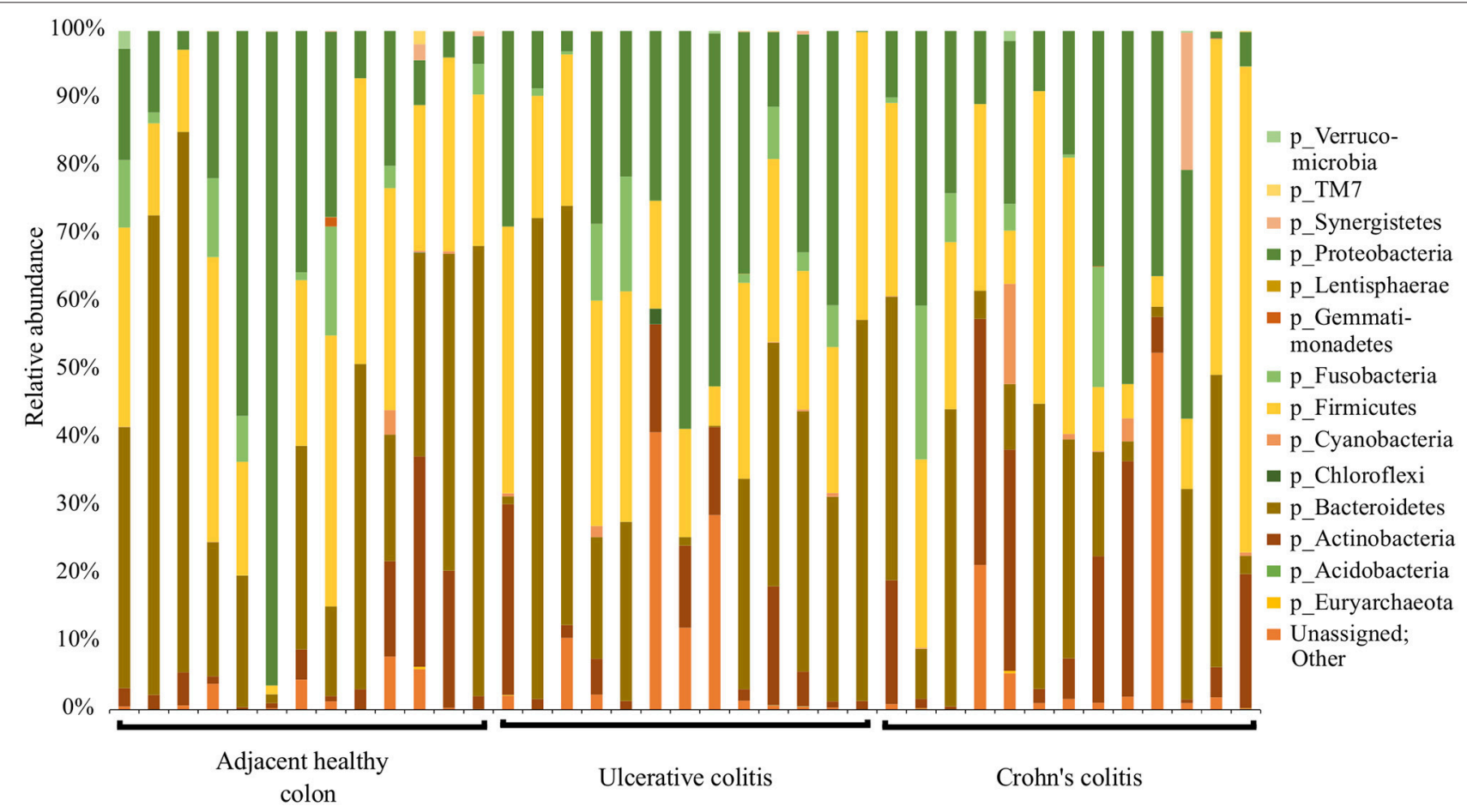

FIGURE 1 | Summary of major bacterial taxa contributing to the communities detected in the 39 specimens at the Phylum level in the colitis and adjacent healthy specimen groups. Specimens are categorized into adjacent healthy colon ( $n=13)$, Ulcerative colitis (UC, $n=13)$, and Crohn's colitis (CC, $n=13)$. Data represented are the relative abundances (\%) of each phylum identified in each specimen. 
TABLE 1 | Functions and proportions of specific pathogenic Oral bacteria colonized in full thickness colon specimens.

\begin{tabular}{|c|c|c|c|c|c|c|}
\hline SI. No. & Bacteria genus & Bacteria species & $\begin{array}{l}\text { Proportion } \\
\text { (\%) }\end{array}$ & Bacteria phylum & Function in IBD & $\begin{array}{l}\text { NCBI genome } \\
\text { database link }\end{array}$ \\
\hline
\end{tabular}

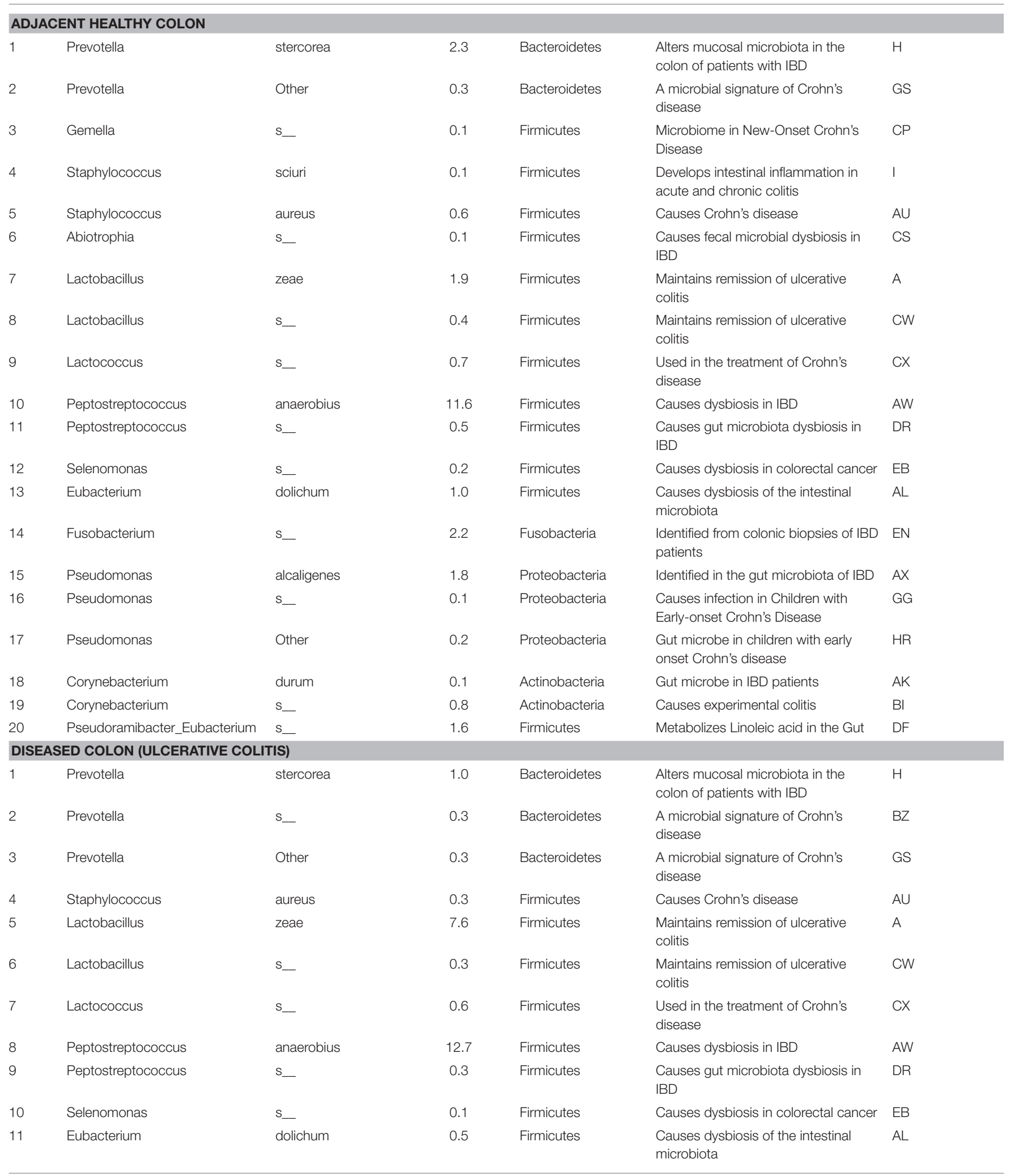


TABLE 1 | Continued

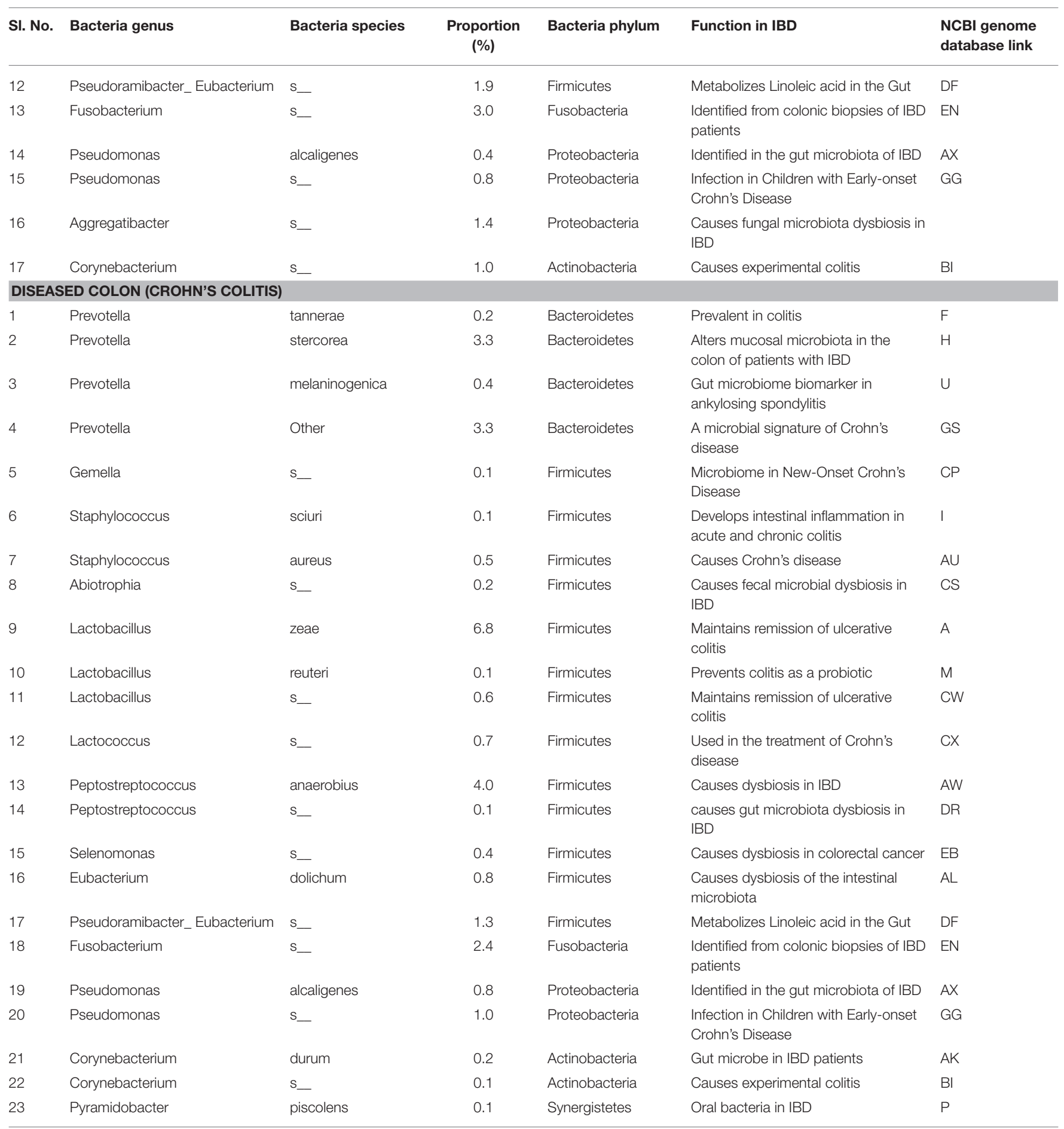

Specific Information of functions was adapted from NCBI Genome Database (https://www.ncbi.n/m.nih.gov/genome/).

The bacterial species that could not be identified at the genus level are mentioned as $g$ _ and the bacterial species that could not be identified at the species level are mentioned as s_.

(27.8\%), and Proteobacteria (24.5\%). Most importantly, our results indicate that putative oral pathogens (belonging to mostly Phylum Firmicutes) dominated the microbiome of diseased specimens (Figure 1). Adjacent healthy specimens show an increased abundance of Phylum Bacteroidetes $(\sim 57 \%$, containing mostly symbiotic and/or beneficial bacteria) population, which is altered in disease categories (Figure 2). 
TABLE 2 | Functions and proportions of specific beneficial Gut bacteria colonized in full thickness colon specimens.

\begin{tabular}{llccc}
\hline SI. No. Bacteria genus & Bacteria species & $\begin{array}{c}\text { Proportion } \\
(\%)\end{array}$ & Bacteria phylum & Function in IBD \\
database link
\end{tabular}

\begin{tabular}{|c|c|c|c|c|c|c|}
\hline \multicolumn{7}{|c|}{ ADJACENT HEALTHY COLON } \\
\hline 1 & Shuttleworthia & satelles & 0.2 & Firmicutes & Identified in the human ileum & J \\
\hline 2 & Bifidobacterium & longum & 0.1 & Actinobacteria & $\begin{array}{l}\text { Attenuates acute murine } \\
\text { experimental model of IBD }\end{array}$ & Y \\
\hline 3 & Rhizobium & leguminosarum & 0.1 & Proteobacteria & $\begin{array}{l}\text { Identified commensal gut } \\
\text { microbe }\end{array}$ & $\mathrm{AB}$ \\
\hline 4 & Lysinibacillus & boronitolerans & 12.1 & Firmicutes & $\begin{array}{l}\text { Identified commensal gut } \\
\text { microbe }\end{array}$ & AT \\
\hline 5 & Alloiococcus & $s_{-}$ & 1.7 & Firmicutes & $\begin{array}{l}\text { Identified commensal gut } \\
\text { microbe }\end{array}$ & CT \\
\hline 6 & Christensenella & S_ & 2.2 & Firmicutes & Identified gut microbe & DC \\
\hline 7 & Blautia & $s_{-}$ & 0.3 & Firmicutes & $\begin{array}{l}\text { Butyrate-producing bacterial } \\
\text { species in Gut }\end{array}$ & $\mathrm{DH}$ \\
\hline 8 & Coprococcus & $s_{-}$ & 0.1 & Firmicutes & $\begin{array}{l}\text { Butyrate-producing bacterial } \\
\text { species in Gut }\end{array}$ & $\mathrm{DI}$ \\
\hline 9 & g_ & $\mathrm{S}_{-}$ & 0.7 & Gemmatimonadetes & $\begin{array}{l}\text { Identified commensal gut } \\
\text { microbe }\end{array}$ & EP \\
\hline 10 & g_ & $s_{-}$ & 0.7 & Lentisphaerae & Normal gut microbe & $\mathrm{EQ}$ \\
\hline 11 & g_ & $s_{-}$ & 0.4 & Proteobacteria & $\begin{array}{l}\text { Identified commensal gut } \\
\text { microbe }\end{array}$ & $\mathrm{FC}$ \\
\hline 12 & Comamonas & $s_{-}$ & 1.0 & Proteobacteria & $\begin{array}{l}\text { Identified commensal gut } \\
\text { microbe }\end{array}$ & $\mathrm{FL}$ \\
\hline 13 & Desulfovibrio & $s_{-}$ & 0.3 & Proteobacteria & Sulfate reducing bacteria in IBD & FV \\
\hline 14 & Paracoccus & Other & 0.1 & Proteobacteria & $\begin{array}{l}\text { Identified commensal gut } \\
\text { microbe }\end{array}$ & $\mathrm{HH}$ \\
\hline 15 & Other & Other & 0.2 & Proteobacteria & Mucosal and fecal microbe & $\mathrm{HP}$ \\
\hline \multicolumn{7}{|c|}{ DISEASED COLON (ULCERATIVE COLITIS) } \\
\hline 1 & Lysinibacillus & boronitolerans & 8.2 & Firmicutes & $\begin{array}{l}\text { Identified commensal gut } \\
\text { microbe }\end{array}$ & AT \\
\hline 2 & Varibaculum & $s_{-}$ & 0.2 & Actinobacteria & $\begin{array}{l}\text { Identified in the gut of a } \\
\text { premature infant }\end{array}$ & $\mathrm{BH}$ \\
\hline 3 & Alloiococcus & $s_{-}$ & 3.1 & Firmicutes & $\begin{array}{l}\text { Identified commensal gut } \\
\text { microbe }\end{array}$ & CT \\
\hline 4 & Christensenella & $s_{-}$ & 0.6 & Firmicutes & Identified gut microbe & DC \\
\hline 5 & Blautia & $s_{-}$ & 0.3 & Firmicutes & $\begin{array}{l}\text { Butyrate-producing bacterial } \\
\text { species in Gut }\end{array}$ & $\mathrm{DH}$ \\
\hline 6 & Coprococcus & $S_{-}$ & 0.2 & Firmicutes & $\begin{array}{l}\text { Butyrate-producing bacterial } \\
\text { species in Gut }\end{array}$ & $\mathrm{DI}$ \\
\hline 7 & g_ & $s_{-}$ & 0.3 & Gemmatimonadetes & $\begin{array}{l}\text { Identified commensal gut } \\
\text { microbe }\end{array}$ & EP \\
\hline 8 & g_ & $s_{-}$ & 0.3 & Proteobacteria & $\begin{array}{l}\text { Identified commensal gut } \\
\text { microbe }\end{array}$ & FC \\
\hline 9 & Comamonas & $s_{-}$ & 2.7 & Proteobacteria & $\begin{array}{l}\text { Identified commensal gut } \\
\text { microbe }\end{array}$ & $\mathrm{FL}$ \\
\hline 10 & Desulfovibrio & S_ & 0.1 & Proteobacteria & Sulfate reducing bacteria in IBD & FV \\
\hline 11 & Morganella & $s_{-}$ & 0.1 & Proteobacteria & Sulfate reducing bacteria in IBD & $\mathrm{FZ}$ \\
\hline 12 & g_ & $S_{-}$ & 0.6 & TM7 & $\begin{array}{l}\text { Identified commensal gut } \\
\text { microbe }\end{array}$ & \\
\hline 13 & Other & Other & 0.1 & Actinobacteria & Commensal gut bacteria in IBD & GN \\
\hline 14 & Other & Other & 0.5 & Proteobacteria & Adult fecal microbe & $\mathrm{HO}$ \\
\hline \multicolumn{7}{|c|}{ DISEASED COLON (CROHN'S COLITIS) } \\
\hline 1 & Akkermansia & muciniphila & 0.1 & Verrucomicrobia & $\begin{array}{l}\text { Adheres to enterocytes and } \\
\text { strengthens the integrity of the } \\
\text { epithelial cell layer }\end{array}$ & S \\
\hline 2 & Bifidobacterium & longum & 0.4 & Actinobacteria & $\begin{array}{l}\text { Attenuates acute murine } \\
\text { experimental model of IBD }\end{array}$ & Y \\
\hline
\end{tabular}


TABLE 2 | Continued

\begin{tabular}{|c|c|c|c|c|c|c|}
\hline SI. No. & Bacteria genus & Bacteria species & $\begin{array}{l}\text { Proportion } \\
(\%)\end{array}$ & Bacteria phylum & Function in IBD & $\begin{array}{l}\text { NCBI genome } \\
\text { database link }\end{array}$ \\
\hline 3 & Rhizobium & leguminosarum & 0.2 & Proteobacteria & $\begin{array}{l}\text { Identified commensal gut } \\
\text { microbe }\end{array}$ & $\mathrm{AB}$ \\
\hline 4 & Anoxybacillus & kestanbolensis & 0.3 & Firmicutes & $\begin{array}{l}\text { Identified commensal gut } \\
\text { microbe }\end{array}$ & $A D$ \\
\hline 5 & Lysinibacillus & boronitolerans & 11.5 & Firmicutes & $\begin{array}{l}\text { Identified commensal gut } \\
\text { microbe }\end{array}$ & AT \\
\hline 6 & g_ & $s_{-}$ & 0.1 & Acidobacteria & $\begin{array}{l}\text { Identified in human gut } \\
\text { microbiota }\end{array}$ & $\mathrm{BC}$ \\
\hline 7 & Varibaculum & S_ & 0.1 & Actinobacteria & $\begin{array}{l}\text { Identified in the gut of a } \\
\text { premature infant }\end{array}$ & $\mathrm{BH}$ \\
\hline 8 & SHD-231 & $\mathrm{s}$ & 0.1 & Chloroflexi & $\begin{array}{l}\text { Identified in the fecal microbiome } \\
\text { of Gout patients }\end{array}$ & $\mathrm{CH}$ \\
\hline 9 & $g_{-}$ & $\mathrm{s}_{-}$ & 0.1 & Cyanobacteria & $\begin{array}{l}\text { Identified in human gut } \\
\text { microbiota }\end{array}$ & CJ \\
\hline 10 & Alloiococcus & s_ & 7.1 & Firmicutes & $\begin{array}{l}\text { Identified commensal gut } \\
\text { microbe }\end{array}$ & CT \\
\hline 11 & Christensenella & $s_{-}$ & 0.1 & Firmicutes & Identified gut microbe & DC \\
\hline 12 & $g_{-}$ & s_ & 0.1 & Firmicutes & Commensal gut bacteria in IBD & DG \\
\hline 13 & Blautia & s_ & 0.1 & Firmicutes & $\begin{array}{l}\text { Butyrate-producing bacterial } \\
\text { species in Gut }\end{array}$ & $\mathrm{DH}$ \\
\hline 14 & Coprococcus & s_ & 0.1 & Firmicutes & $\begin{array}{l}\text { Butyrate-producing bacterial } \\
\text { species in Gut }\end{array}$ & $\mathrm{Dl}$ \\
\hline 15 & g_ & $s_{-}$ & 0.3 & Gemmatimonadetes & $\begin{array}{l}\text { Identified commensal gut } \\
\text { microbe }\end{array}$ & EP \\
\hline 16 & g_ & s_ & 0.3 & Lentisphaerae & Normal gut microbe & $E Q$ \\
\hline 17 & Comamonas & $\mathrm{s}$ & 2.4 & Proteobacteria & $\begin{array}{l}\text { Identified commensal gut } \\
\text { microbe }\end{array}$ & FL \\
\hline 18 & $g_{-}$ & $\mathrm{s}_{-}$ & 0.1 & Proteobacteria & $\begin{array}{l}\text { Identified commensal gut } \\
\text { microbe }\end{array}$ & $\mathrm{FT}$ \\
\hline 19 & Desulfovibrio & $s_{-}$ & 0.2 & Proteobacteria & Sulfate reducing bacteria in IBD & FV \\
\hline 20 & g_ & $s_{-}$ & 0.3 & TM7 & $\begin{array}{l}\text { Identified commensal gut } \\
\text { microbe }\end{array}$ & \\
\hline 21 & Other & Other & 0.2 & Firmicutes & Commensal gut bacteria in IBD & $\mathrm{HA}$ \\
\hline 22 & Paracoccus & Other & 0.7 & Proteobacteria & $\begin{array}{l}\text { Identified commensal gut } \\
\text { microbe }\end{array}$ & $\mathrm{HH}$ \\
\hline 23 & Other & Other & 0.1 & Proteobacteria & $\begin{array}{l}\text { Identified commensal gut } \\
\text { microbe }\end{array}$ & $\mathrm{HN}$ \\
\hline 24 & Other & Other & 0.7 & Proteobacteria & Adult fecal microbe & $\mathrm{HO}$ \\
\hline
\end{tabular}

Specific Information of functions was adapted from NCBI Genome Database (https://www.ncbi.nlm.nih.gov/genome/).

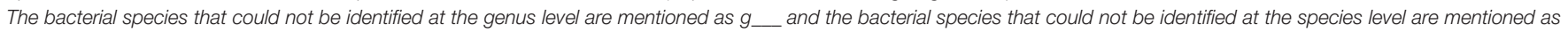
s_.

\section{Differential Expression of Microbiomes in the Colon of CA and AA Patients}

Figure 3 show racial differences of various bacterial phyla in adjacent healthy, UC and CC full thickness colon specimens. The tissue specimens from Caucasians represented a significantly higher proportion $(p<0.05)$ of the oral pathogen, Fusobacterium, and gut bacteria, Parabacteroides (Bacteroidetes). CA specimens also showed significantly higher levels $(p<0.05)$ of Phyla Proteobacteria including Citrobacter, Hemophilus, Acinetobacter, Pseudomonas, and Stenotrophomonas as compared to AA. Whereas, the AA specimens were observed to have a significantly higher proportion $(p<0.05)$ of Prevotella (Bacteroidetes) and Clostridia (Firmicutes) (Figure 3; Table 4).

As depicted in Figure 3, the adjacent healthy colon specimens, UC and CC contained $\sim 1 \%, \sim 7 \%$ and $\sim 7 \%$ of sequence reads, respectively that were un-assignable to any taxon with a larger proportion of them identified in AA Colitis patients. Other major phyla observed among these specimens also include Proteobacteria (Adjacent healthy: 23.8\%; UC: $26.5 \%$ and CC: 23.1\%), Actinobacteria (Adjacent healthy: 6.7\%; UC: $8.1 \%$ and CC: $14.1 \%$ ), Fusobacteria (Adjacent healthy: $4.2 \%$; UC: $3.6 \%$ and CC: $4.0 \%$ ), and Synergistetes (Adjacent healthy: 0.2\%; UC: $0.04 \%$ and CC: $1.5 \%$ ). The Phylum Proteobacteria did not show 
TABLE 3 | Functions and proportions of specific pathogenic Gut bacteria colonized in full thickness colon specimens.

\section{SI. No. Bacteria genus Bacteria species Proportion Bacteria phylum Function in IBD}

(\%)
NCBI Genome

database link

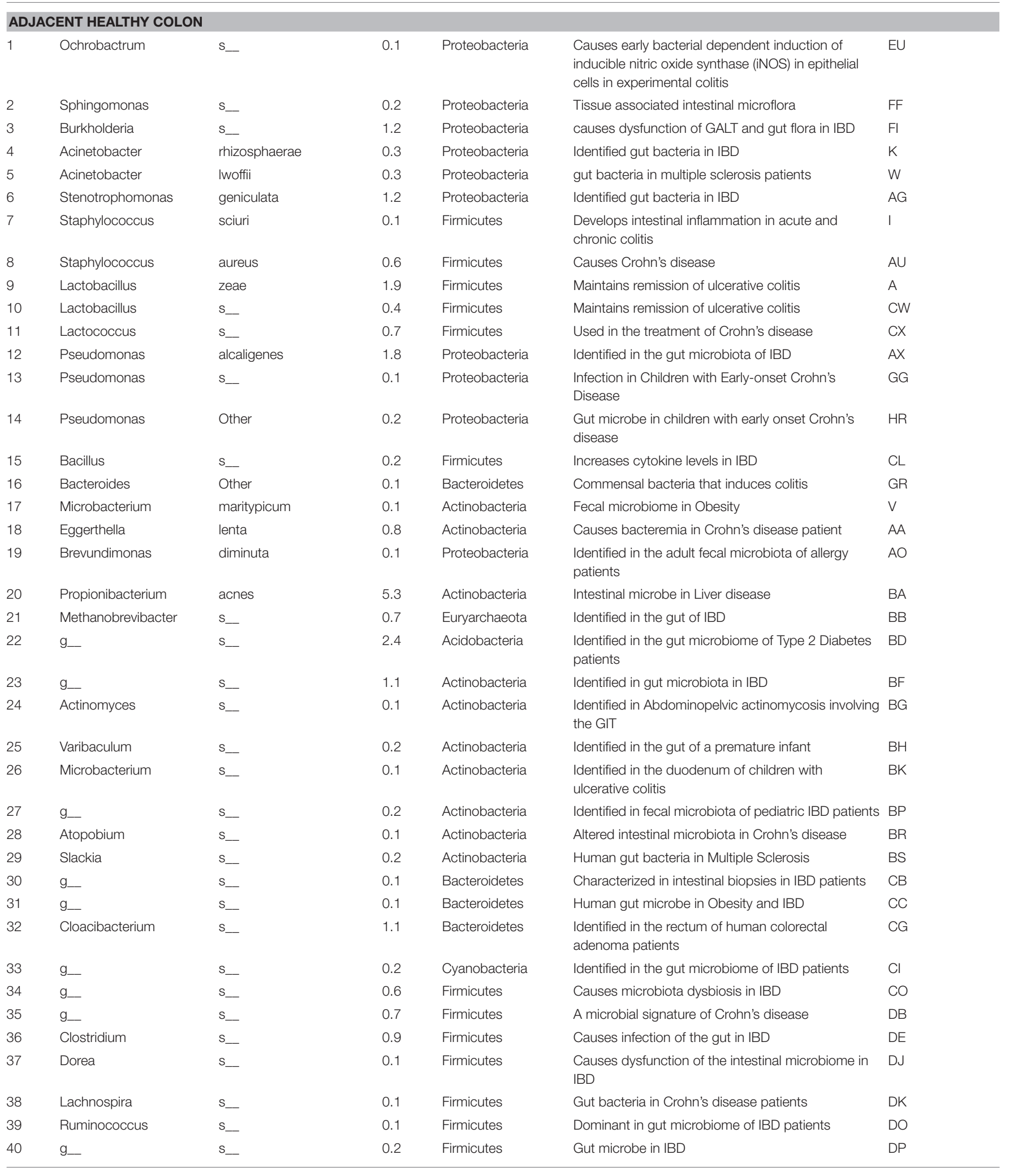


TABLE 3 | Continued

\section{SI. No. Bacteria genus}

Bacteria species

Proportion Bacteria phylum

(\%)

\begin{tabular}{lll}
\hline 41 & g__ & S \\
42 & Anaerotruncus & $\mathrm{s}$ \\
43 & Oscillospira & $\mathrm{s}$ \\
44 & Ruminococcus & $\mathrm{s}$ \\
45 & g_ & $\mathrm{s}$ \\
46 & Acidaminococcus & $\mathrm{s}$ \\
47 & Phascolarctobacterium
\end{tabular}

48

49

50

51

52

53

54 Coprobacillus

55 Leptotrichia

56 g_

57

58

59 9_

60 Lautropia

61 g_

62 Citrobacter

63 Halomonas

64 g_

65 g_

66 Other

67 Other

68 Other

69 Weissella

70 Other

71 Other

DISEASED COLON (ULCERATIVE COLITIS)

0.6

0.6

0.2

0.4

0.7

0.4

1.3

1.9

0.5

0.2

1.3

0.3

0.3

0.1

0.3

0.7

4.2

0.1

0.1

1.2

0.3

0.1

0.1

1.4

0.1

0.1

0.1

3.6

12.5

0.2

0.1

0.1

(2)

Firmicutes
Firmicutes
Firmicutes
Firmicutes
Firmicutes
Firmicutes
Firmicutes

Firmicutes

Firmicutes

Firmicutes

Firmicutes

Firmicutes

Firmicutes

Firmicutes

Fusobacteria

Proteobacteria

Proteobacteria

Proteobacteria

Proteobacteria

Proteobacteria

Proteobacteria

Proteobacteria

Proteobacteria

Proteobacteria

Proteobacteria

Actinobacteria

Firmicutes

Firmicutes

Firmicutes

Proteobacteria

Proteobacteria
S

S__
s__
s__
rhizosphaerae
Iwoffii
s__
geniculata
s__

punction in IBD

$0.1 \quad$ Proteobacteria

Proteobacteria

Proteobacteria

Proteobacteria

Proteobacteria

Proteobacteria

Proteobacteria

Proteobacteria

Firmicutes

Firmicutes

Function in IBD

A microbial signature of Crohn's disease

Tissue associated intestinal microflora

Gut microbe in IBD patients

Dominant in gut microbiome of IBD patients

Gut microbe underlying the onset of IBD

Gut microbe in IBD

IBD

causes fecal microbial dysbiosis in IBD

A microbial signature of Crohn's disease

Microbe in Inflammatory Pouch Complications

Intestinal microbe in colorectal cancer

Gut microbe in $\mathrm{Gl}$ diseases

Fecal-associated and mucosalassociated

microbiota in irritable bowel syndrome patients

Alters Gut Microbiota in Psoriatic Arthritis

Causes gut mucosal inflammation in Rheumatoid arthritis patients

Intestinal microbe in children with severe and EV

complicated acute viral gastroenteritis

Causes microbial dysbiosis in pediatric Crohn's EW

disease

Intestinal microbe in children with severe and EX

complicated acute viral gastroenteritis

Involved in host-microbial cross talk in IBD

causes fecal microbial dysbiosis in IBD

Fecal and mucosa associated microbe in IBD

Gut microbe in newly diagnosed with

treatment-naïve Crohn's disease patients

Intestinal microflora in chronic kidney disease

Microbe in colon tissue from IBD subjects

bacteria in human Ulcerative Colitis patients

Alters fecal microbiota in pediatric IBD patients

gut microbe in experimental colitis

Fecal and mucosa associated microbe in IBD

Gut microbe in IBD patients

Fecal and mucosa associated microbe in IBD

Involved in host-microbial cross talk in IBD

Causes early bacterial dependent induction of

inducible nitric oxide synthase (iNOS) in epithelial

cells in experimental colitis

Fecal and mucosa associated microbe in IBD FM

Tissue associated intestinal microflora FF

Causes dysfunction of GALT and gut flora in IBD FI

Identified gut microbe in IBD

Gut bacteria in multiple sclerosis patients

Tissue associated intestinal microflora

Identified gut microbe in IBD

Induces experimental IBD

Develops intestinal inflammation in acute and

chronic colitis
NCBI genome

database link

DQ

DT

DU

DV

DW

DX

EA

EC

EE

EF

El

EJ

EL

EO

EV

N

X

FJ

FK

FY

GB

GE

$\mathrm{GH}$

GO

GT

GW

GX

HL

$\mathrm{HM}$

EU

K

WF

AG

CV

I 
TABLE 3 | Continued

\section{SI. No. Bacteria genus}

\begin{tabular}{ll}
\hline 11 & Staphylococcus \\
12 & Lactobacillus \\
13 & Lactobacillus \\
14 & Lactococcus \\
15 & Pseudomonas \\
16 & Pseudomonas
\end{tabular}

17

18

19

20

21

22

23

24

25
Pseudomonas

\section{Bacillus}

Bacteroides

Bacteroides

Blautia

Faecalibacterium

Microbacterium

Eggerthella

Propionibacterium

Methanobrevibacter

g_

\section{g_}

Adlercreutzia

Slackia

g_

g_

g_

Cloacibacterium

g_

g_

g_

Clostridium

Dorea

\section{Lachnospira}

Ruminococcus

g_

Oscillospira

Ruminococcus

g_

Acidaminococcus

Phascolarctobacterium

\section{Schwartzia}

Anaerococcus

Finegoldia

g_

Bulleidia

Coprobacillus

Leptotrichia
Bacteria species

Proportior
(\%)

aureus
zeae
s__
s__
alcaligenes
Other

s_

caccae

Other

producta

prausnitzii

maritypicum

lenta

acnes

$\mathrm{s}$

s

S

s_

s_

S_

$\mathrm{S}$

$\mathrm{S}$

S_

S

S

s_

s_

S

S

s_

$\mathrm{S}$

$\mathrm{s}$

s_

s_

S-

S

-

$\mathrm{s}$

S

$\mathrm{S}$

$\mathrm{s}$

S

S_

$\mathrm{s}$

0.6

0.6
1.9

1.9

0.4

0.7

1.8

0.1

0.2

0.1

0.1

0.2

0.1

0.1

0.1

5.1

2.8

0.3

1.8

0.3

0.2

0.2

0.8

0.4

0.1

0.4

0.1

0.1

1.7

0.8

0.1

0.3

0.1

0.3

0.1

0.9

0.3

1.1

2.2

0.5

0.6

0.3

0.6

0.1

0.1

0.5
Function in IBD

Bacteria phylu

Firmicutes

Firmicutes

Firmicutes

Firmicutes

Proteobacteria

Proteobacteria

Proteobacteria

Firmicutes

Bacteroidetes

Bacteroidetes

Firmicutes

Firmicutes

Actinobacteria

Actinobacteria

Actinobacteria

Euryarchaeota

Acidobacteria

Actinobacteria

Actinobacteria

Actinobacteria

Bacteroidetes

Bacteroidetes

Bacteroidetes

Bacteroidetes

Firmicutes

Firmicutes

Firmicutes

Firmicutes

Firmicutes

Firmicutes

Firmicutes

Firmicutes

Firmicutes

Firmicutes

Firmicutes

Firmicutes

Firmicutes

Firmicutes

Firmicutes

Firmicutes

Firmicutes

Firmicutes

Firmicutes

Fusobacteria

Disease disease patients IBD IBD

\section{Causes Crohn's disease}

Maintains remission of ulcerative colitis

Maintains remission of ulcerative colitis

used in the treatment of Crohn's disease

Identified in the gut microbiota of IBD

Infection in Children with Early-onset Crohn's

Gut microbe in children with early onset Crohn's HR

Increases cytokine levels in IBD

Identified in the gut of ulcerative colitis patients

Commensal bacteria that induces colitis

Gut microbe in Obesity and IBD

Gut microbe in Crohn's disease patients

Fecal microbiome in Obesity

Causes bacteremia in Crohn's disease patient

Intestinal microbe in Liver disease

Identified in the gut of IBD

NCBI genome

database link

Identified in the gut microbiome of Type 2 Diabetes

Identified in gut microbiota in IBD BF

Causes dysbiosis in IBD patients $\quad B Q$

Alters human gut microbiome in Multiple Sclerosis BS

Identified in gut microbiome of IBD patients CA

Characterized in intestinal biopsies in IBD patients $\mathrm{CB}$

Human gut microbe in Obesity and IBD CC

Identified in the rectum of human colorectal CG

adenoma patients

Causes microbiota dysbiosis in IBD $\quad \mathrm{CO}$

Gut microbe in IBD DA

A microbial signature of Crohn's disease DB

Causes infection of the gut in IBD DE

Causes dysfunction of the intestinal microbiome in DJ

gut bacteria in Crohn's disease patients DK

Dominant in gut microbiome of IBD patients DO

A microbial signature of Crohn's disease $D Q$

Gut microbe in IBD patients DU

Dominant in gut microbiome of IBD patients DV

Gut microbe underlying the onset of IBD DW

Gut microbe in IBD DX

Causes dysfunction of the intestinal microbiome in DZ

Causes fecal microbial dysbiosis in IBD EA

Microbe in Inflammatory Pouch Complications EE

Intestinal microbe in colorectal cancer EF

Gut microbe in Gl diseases El

Fecal-associated and mucosalassociated EJ

microbiota in irritable bowel syndrome patients

Alters Gut Microbiota in Psoriatic Arthritis

EL

Causes gut mucosal inflammation in Rheumatoid EO arthritis patients 
TABLE 3 | Continued

SI. No. Bacteria genus

Bacteria species

Proportion Bacteria phylum

\begin{tabular}{|c|c|c|}
\hline 55 & g_ & s_- \\
\hline 56 & g_ & $\mathrm{s}$ \\
\hline 57 & g_- & s_ \\
\hline 58 & g_ & s_ \\
\hline 59 & Sutterella & $s_{-}$ \\
\hline 60 & Lautropia & s_ \\
\hline 61 & g_ & $\mathrm{s}_{-}$ \\
\hline 62 & Citrobacter & s_- \\
\hline 63 & Halomonas & s_ \\
\hline 64 & $g_{-}$ & $s_{-}$ \\
\hline 65 & Other & Other \\
\hline 66 & Eggerthella & Other \\
\hline 67 & Other & Other \\
\hline 68 & Other & Other \\
\hline 69 & Weissella & Other \\
\hline 70 & Other & Other \\
\hline 71 & Other & Other \\
\hline \multicolumn{3}{|c|}{ DISEASED COLON (CROHN'S COLITIS) } \\
\hline
\end{tabular}

Ochrobactrum

Sphingomonas

Burkholderia

Acinetobacter

Acinetobacter

Acinetobacter

Stenotrophomonas

Enterococcus

Staphylococcus

Staphylococcus

Lactobacillus

Lactobacillus

Lactococcus

Pseudomonas

Pseudomonas

Pseudomonas

Bacillus

Bacillus

Bacteroides

Bacteroides

Microbacterium

Eggerthella

Brevundimonas s_

S_

3.6 Proteobacteria

$0.5 \quad$ Proteobacteria

0.1 Proteobacteria

1.5 Proteobacteria

0.1 Proteobacteria

0.2 Proteobacteria

0.5 Proteobacteria

0.3 Proteobacteria

$0.8 \quad$ Proteobacteria

0.3 Proteobacteria

0.2 Actinobacteria

0.1 Actinobacteria

4.0 Firmicutes

$4.2 \quad$ Firmicutes

1.2 Firmicutes

2.5 Proteobacteria

1.4 Proteobacteria

0.1

Proteobacteria

Proteobacteria

Proteobacteria

Proteobacteria

Proteobacteria

Proteobacteria

geniculata

s_

sciuri

aureus

zeae

$\mathrm{s}$

$\mathrm{s}$

alcaligenes

$\mathrm{s}$

Other

thermoamylovorans

s_

eggerthii

Other

maritypicum

lenta

diminuta

Proteobacteria

Firmicutes

Firmicutes

Firmicutes

Firmicutes

Firmicutes

Firmicutes

Proteobacteria

Proteobacteria

Proteobacteria

0.2

Firmicutes

Firmicutes

Bacteroidetes

Bacteroidetes

Actinobacteria

Actinobacteria

Proteobacteria
Iwoffii
(\%)

Function in IBD

Intestinal microbe in children with severe and complicated acute viral gastroenteritis

Causes chronic inflammation in IBD

Microbial factor associated with postoperative

Crohn's disease

Involved in host-microbial cross talk in IBD

Gut microbe in experimental colitis

Causes fecal microbial dysbiosis in IBD

Fecal and mucosa associated microbe in IBD

Gut microbe in newly diagnosed with

treatment-naïve Crohn's disease patients

Intestinal microflora in chronic kidney disease

Bacteria in human Ulcerative Colitis patients

Alters fecal microbiota in pediatric IBD patients

Causes bacteremia in Crohn's disease patient

Gut microbe in experimental colitis

Fecal and mucosa associated microbe in IBD

Gut microbe in IBD patients

Causes microbial dysbiosis in pediatric Crohn's

disease

Fecal and mucosa associated microbe in IBD

Causes early bacterial dependent induction of inducible nitric oxide synthase (iNOS) in epithelial cells in experimental colitis

Tissue associated intestinal microflora

Causes dysfunction of GALT and gut flora in IBD

Identified gut microbe in IBD

Gut bacteria in multiple sclerosis patients

Tissue associated intestinal microflora in colitis patients

Identified gut microbe in IBD

Induces experimental IBD

Develops intestinal inflammation in acute and chronic colitis

Causes Crohn's disease

Maintains remission of ulcerative colitis

Maintains remission of ulcerative colitis

Used in the treatment of Crohn's disease

Identified in the gut microbiota of IBD

Infection in Children with Early-onset Crohn's

Disease

Gut microbe in children with early onset Crohn's disease
A probiotic- normal flora of the gut

Increases cytokine levels in IBD

Enhances colitis in mice

Commensal bacteria that induces colitis

Fecal microbiome in Obesity

Causes bacteremia in Crohn's disease patient

Identified in the adult fecal microbiota of allergy patients
NCBI genome

database link

EV

FB

FD

$F G$

$\mathrm{FH}$

FJ

FK

FY

GB

$\mathrm{GH}$

GO

GQ

GT

GW

GX

$H D$

$\mathrm{HL}$

EU

$\mathrm{FI}$

K

W

$\mathrm{HQ}$

AG

CV

I

$\mathrm{AU}$

A

CW

CX

AX

GG

$\mathrm{HR}$

E

CL

AJ

GR

V

AA

AO 
TABLE 3 | Continued

\section{SI. No. Bacteria genus}

Bacteria species

Propo

(\%)

\begin{tabular}{lll}
\hline 24 & Propionibacterium & acnes \\
25 & Methanobrevibacter & S_ \\
26 & g_ & s_
\end{tabular}

28

29

g_

Microbacterium

S-

S_

S

Bifidobacterium

g_

Atopobium

Slackia

g_

g_

g-

g_

g_

Clostridium

Lachnospira

Moryella

g_

g_

Oscillospira

Ruminococcus

g_

Acidaminococcus

Phascolarctobacterium

Schwartzia

g_

Anaerococcus

Finegoldia

g_

Bulleidia

55 Coprobacillus

$56 \quad$ Leptotrichia

57 g_

$58 \quad$ g_

59 g_

60 Sutterella

61 Lautropia

62 g_

63 g_

64 Ralstonia

65 Halomonas

1.2 Actinobacteria

0.8 Euryarchaeota

0.7 Acidobacteria

0.2 Actinobacteria

0.5 Actinobacteria

0.1 Actinobacteria

0.2 Actinobacteria

0.3 Actinobacteria

0.2 Actinobacteria

1.3 Actinobacteria

0.1 Bacteroidetes

0.3 Bacteroidetes

$0.7 \quad$ Firmicutes

0.2 Firmicutes

0.9 Firmicutes

0.6 Firmicutes

0.6 Firmicutes

$0.1 \quad$ Firmicutes

0.1 Firmicutes

0.6 Firmicutes

0.2 Firmicutes

0.9 Firmicutes

0.2 Firmicutes

1.0 Firmicutes

0.6 Firmicutes

0.2

0.1

0.3

0.4

0.5

0.2

0.3

0.5

4.1

0.2

1.2

0.1

0.1

0.4

0.1

0.1

0.5

Firmicutes

Firmicutes

Firmicutes

Firmicutes

Firmicutes

Firmicutes

Firmicutes

Fusobacteria

Proteobacteria

Proteobacteria

Proteobacteria

Proteobacteria

Proteobacteria

Proteobacteria

Proteobacteria

Proteobacteria
Proteobacteria
Function in IBD

Intestinal microbe in Liver disease

Identified in the gut of IBD patients

Identified in the gut microbiome of Type 2 Diabetes BD patients

Identified in gut microbiota in IBD

Identified in gut microbiota in IBD

Identified in the duodenum of children with ulcerative colitis

Identified in gut microbiota of IBD patients $\quad B O$

Identified in fecal microbiota of pediatric IBD patients BP

Altered intestinal microbiota in Crohn's disease BR

Alters human gut microbiome in Multiple Sclerosis BS

Identified in gut microbiome of IBD patients CA

Human gut microbe in Obesity and IBD CC

Causes microbiota dysbiosis in IBD CO

Gut microbe in IBD

A microbial signature of Crohn's disease

Causes infection of the gut in IBD

Gut bacteria in Crohn's disease patients

Microbe in Inflammatory Pouch Complications

gut microbe in IBD

A microbial signature of Crohn's disease

Gut microbe in IBD patients

Dominant in gut microbiome of IBD patients

Gut microbe underlying the onset of IBD

Gut microbe in IBD IBD

Causes fecal microbial dysbiosis in IBD

A microbial signature of Crohn's disease

Microbe in Inflammatory Pouch Complications

Intestinal microbe in colorectal cancer

Gut microbe in Gl diseases

Fecal-associated and mucosalassociated microbiota in irritable bowel syndrome patients

Alters Gut Microbiota in Psoriatic Arthritis

NCBI genome

database link

A
B
BE

BF

BK

O
S
C
DA

DA
DB

DE

DK

DL

DP

DQ

DU

DV

DW

DX

DZ

EA

EC

EE

EF

El

EJ

EL

Causes gut mucosal inflammation in Rheumatoid EO arthritis patients

Intestinal microbe in children with severe and EV

complicated acute viral gastroenteritis

Microbial factor associated with postoperative FD

Crohn's disease

Involved in host-microbial cross talk in IBD FG

Gut microbe in experimental colitis $\mathrm{FH}$

Causes fecal microbial dysbiosis in IBD FJ

Fecal and mucosa associated microbe in IBD FK

Bacteria in Mucosal and Submucosal Intestinal FN

Tissues in Advanced Crohn's Disease

Microbiota in the Mucosa of Patients With Ulcerative FP

Colitis

Intestinal microflora in chronic kidney disease

GB 
TABLE 3 | Continued

\begin{tabular}{|c|c|c|c|c|c|c|}
\hline SI. No. & Bacteria genus & Bacteria species & $\begin{array}{l}\text { Proportion } \\
\text { (\%) }\end{array}$ & Bacteria phylum & Function in IBD & $\begin{array}{l}\text { NCBI genome } \\
\text { database link }\end{array}$ \\
\hline 66 & Haemophilus & s_ & 0.7 & Proteobacteria & $\begin{array}{l}\text { Treatment naïve microbiome in new onset Crohn's } \\
\text { disease }\end{array}$ & GD \\
\hline 67 & g_ & s_ & 0.1 & Proteobacteria & Microbe in colon tissue from IBD subjects & GE \\
\hline 68 & Other & Other & 1.1 & Actinobacteria & Alters fecal microbiota in pediatric IBD patients & GO \\
\hline 69 & Eggerthella & Other & 0.1 & Actinobacteria & Causes bacteremia in Crohn's disease patient & GQ \\
\hline 70 & Other & Other & 1.7 & Firmicutes & Gut microbe in experimental colitis & GT \\
\hline 71 & Other & Other & 2.2 & Firmicutes & Fecal and mucosa associated microbe in IBD & GW \\
\hline 72 & Weissella & Other & 2.2 & Firmicutes & Gut microbe in IBD patients & GX \\
\hline 73 & Other & Other & 0.1 & Proteobacteria & $\begin{array}{l}\text { Causes microbial dysbiosis in pediatric Crohn's } \\
\text { disease }\end{array}$ & $\mathrm{HD}$ \\
\hline 74 & Methylobacterium & Other & 0.1 & Proteobacteria & $\begin{array}{l}\text { Causes gut microbial dysbiosis in pediatric Crohn's } \\
\text { disease patients }\end{array}$ & $H G$ \\
\hline 75 & Other & Other & 0.2 & Proteobacteria & Fecal and mucosa associated microbe in IBD & HK \\
\hline 76 & Other & Other & 0.6 & Proteobacteria & Fecal and mucosa associated microbe in IBD & $\mathrm{HL}$ \\
\hline
\end{tabular}

Specific Information of functions was adapted from NCBI Genome Database (https://www.ncbi.n/m.nih.gov/genome/).

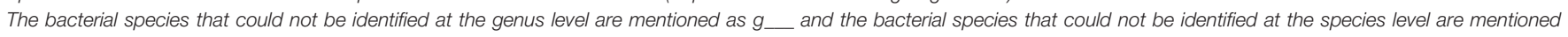
as s_.

any significant difference between healthy colon specimens and diseased colon specimens (Table 4).

\section{Bacterial Species Identified in a Significantly Higher Proportion in Diseased Colon Tissues}

As shown in Figure 3, diseased colon specimens represented a significantly higher proportion $(p<0.05)$ of gut bacteria belonging to Phylum Firmicutes including Blautia producta, Faecalibacterium prausnitzii, Anoxybacillus kestanbolensis, Ruminococcus gnavus, Eubacterium dolichum, Lysinibacillus boronitolerans, and oral bacteria including Staphylococcus sciuri, Staphylococcus aureus, Streptococcus anginosus.

In contrast, healthy colon specimens were significantly dominated ( $p<0.05$ ) by oral bacteria belonging to Phylum Actinobacteria that includes; Corynebacterium kroppenstedtii, Corynebacterium durum. Additionally, healthy colon specimens were dominated by gut bacteria belonging to Phylum Actinobacteria that includes; Colinsella stercoris, Colinsella aerofaciens, Kocuria rhizophila, Eggerthella lenta, Propionibacterium granulosum, Propionibacterium acnes, Actinomyces europaeus, Rothia dentocariosa, and Phylum Bacteroidetes that includes; Bacteroides fragilis, Bacteroides eggerthii, Bacteroides caccae, Parabacteroides distasonis (Figure 3).

\footnotetext{
Alpha Diversity and Beta Diversity Analyses Alpha diversity and beta diversity metrics were computed to analyse the diversity of bacterial species within each sample and between samples. To assess our sampling efficiency, we plotted rarefaction curves (Chaol and Shannon) for all 39 specimens. Increased diversity (Shannon) in the diseased samples compared to control samples was observed. From the rarefaction curves,
}

it is evident that most $\mathrm{AA}$ samples require additional sampling whereas Caucasian samples do not (data not shown).

Since, outliers exhibiting different microbiome profiles were observed both in the healthy and disease groups, we performed principle coordinate analysis (PCoA analysis) and hierarchial clustering to obtain a holistic view of the microbiome profile in each sample. Two dimensional PCoA plots revealed that control samples which had similar microbiome profiles as suggested by histograms and OTU heat map clustered together (data not shown).

\section{Pathogenic Oral and Gut Flora Abundantly Colonized in Diseased Colon Specimens}

The pathogenic oral bacteria identified abundantly in diseased colon specimens as compared to healthy colon specimens were Porphyromonas, Prevotella, Gemella, Staphylococcus, Streptococcus, Abiotrophia, Granulicatella, Lactobacillus, Lactococcus, Peptostreptococcus, Selenomonas, Veillonella, Parvimonas, Eubacterium, Fusobacterium, Pseudomonas, Aggregatibacter, and Corynebacterium (Table 1).

Pathogenic gut bacteria identified abundantly in diseased colon specimens as compared to healthy colon specimens include Ochrobactrum, Delftia, Sphingomonas, Burkholderia, Acinetobacter, Stenotrophomonas, Enterococcus, Granulicatella, Staphylococcus, Streptococcus, Lactobacillus, Lactococcus, Pseudomonas, Bacillus, Campylobacter, and Bacteroides (Table 3).

\section{DISCUSSION}

Our study demonstrates significant perturbations among bacteria belonging to Phyla Bacteroidetes and Firmicutes in fullthickness diseased colon specimens containing neuromuscular compartment (Figure 2). Our studies further show that the proportion of pathogenic bacteria are higher in diseased 
TABLE 4 | Functions and Proportions of bacterial species identified in the full thickness human colon specimens of Caucasians and African Americans.

\begin{tabular}{lcccc}
\hline SI. No. Bacteria genus & Bacteria species & $\begin{array}{c}\text { Proportion } \\
(\%)\end{array}$ & $\begin{array}{c}\text { Bacteria phylum } \\
\text { Function in IBD } \\
\text { database link }\end{array}$
\end{tabular}

\begin{tabular}{|c|c|c|c|c|c|c|}
\hline \multicolumn{7}{|c|}{ CAUCASIAN AMERICANS } \\
\hline 1 & Lactobacillus & zeae & 6.8 & Firmicutes & Maintains remission of ulcerative colitis & A \\
\hline 2 & Bacillus & thermoamylovorans & 0.1 & Firmicutes & A probiotic- normal flora of the gut & $\mathrm{E}$ \\
\hline 3 & Prevotella & tannerae & 0.1 & Bacteroidetes & Prevalent in colitis & $\mathrm{F}$ \\
\hline 4 & Collinsella & stercoris & 0.0 & Actinobacteria & Used for treatment of IBD & $\mathrm{G}$ \\
\hline 5 & Prevotella & stercorea & 1.4 & Bacteroidetes & $\begin{array}{l}\text { Alters mucosal microbiota in the colon of patients } \\
\text { with IBD }\end{array}$ & $\mathrm{H}$ \\
\hline 6 & Staphylococcus & sciuri & 0.1 & Firmicutes & $\begin{array}{l}\text { Develops intestinal inflammation in acute and } \\
\text { chronic colitis }\end{array}$ & 1 \\
\hline 7 & Shuttleworthia & satelles & 0.0 & Firmicutes & Identified in the human ileum & $J$ \\
\hline 8 & Acinetobacter & rhizosphaerae & 1.1 & Proteobacteria & Identified gut microbe in IBD & $\mathrm{K}$ \\
\hline 9 & Blautia & producta & 0.1 & Firmicutes & Gut microbe in Obesity and IBD & $\mathrm{N}$ \\
\hline 10 & Akkermansia & muciniphila & 0.1 & Verrucomicrobia & $\begin{array}{l}\text { Adheres to enterocytes and strengthens the } \\
\text { integrity of the epithelial cell layer }\end{array}$ & S \\
\hline 11 & Prevotella & melaninogenica & 0.2 & Bacteroidetes & Gut microbiome biomarker in ankylosing spondylitis & $U$ \\
\hline 12 & Acinetobacter & Iwoffii & 0.2 & Proteobacteria & Gut bacteria in multiple sclerosis patients & W \\
\hline 13 & Bifidobacterium & longum & 0.2 & Actinobacteria & Attenuates acute murine experimental model of IBD & Y \\
\hline 14 & Eggerthella & lenta & 4.3 & Actinobacteria & Causes bacteremia in Crohn's disease patient & AA \\
\hline 15 & Rhizobium & leguminosarum & 0.1 & Proteobacteria & Identified gut microbe in IBD patients & $A B$ \\
\hline 16 & Anoxybacillus & kestanbolensis & 0.1 & Firmicutes & Identified gut microbe in IBD patients & $A D$ \\
\hline 17 & Stenotrophomonas & geniculata & 0.1 & Proteobacteria & Identified gut microbe in IBD patients & AG \\
\hline 18 & Corynebacterium & durum & 0.1 & Actinobacteria & Identified gut microbe in IBD patients & AK \\
\hline 19 & Eubacterium & dolichum & 0.8 & Firmicutes & Causes dysbiosis of the intestinal microbiota & $\mathrm{AL}$ \\
\hline 20 & Brevundimonas & diminuta & 0.1 & Proteobacteria & $\begin{array}{l}\text { Identified in the adult fecal microbiota of allergy } \\
\text { patients }\end{array}$ & $\mathrm{AO}$ \\
\hline 21 & Lysinibacillus & boronitolerans & 12.3 & Firmicutes & Identified gut microbe in IBD patients & AT \\
\hline 22 & Staphylococcus & aureus & 0.6 & Firmicutes & Causes Crohn's disease & $\mathrm{AU}$ \\
\hline 23 & Peptostreptococcus & anaerobius & 4.8 & Firmicutes & Causes dysbiosis in IBD & AW \\
\hline 24 & Pseudomonas & alcaligenes & 1.0 & Proteobacteria & Identified in the gut microbiota of IBD & $A X$ \\
\hline 25 & Propionibacterium & acnes & 3.9 & Actinobacteria & Intestinal microbe in Liver disease & $\mathrm{BA}$ \\
\hline 26 & Methanobrevibacter & $s_{-}$ & 0.6 & Euryarchaeota & Identified in the gut of IBD & $\mathrm{BB}$ \\
\hline 27 & g_ & $s_{-}$ & 0.8 & Acidobacteria & $\begin{array}{l}\text { Identified in the gut microbiome of Type } 2 \text { Diabetes } \\
\text { patients }\end{array}$ & $\mathrm{BD}$ \\
\hline 28 & $g_{-}$ & $s_{-}$ & 0.9 & Actinobacteria & Identified in gut microbiota in IBD & $\mathrm{BF}$ \\
\hline 29 & Varibaculum & s_ & 0.1 & Actinobacteria & Identified in the gut of a premature infant & $\mathrm{BH}$ \\
\hline 30 & Corynebacterium & S_ & 0.7 & Actinobacteria & Causes experimental colitis & $\mathrm{Bl}$ \\
\hline 31 & Microbacterium & s_ & 0.1 & Actinobacteria & $\begin{array}{l}\text { Identified in the duodenum of children with } \\
\text { ulcerative colitis }\end{array}$ & BK \\
\hline 32 & Bifidobacterium & $s_{-}$ & 0.1 & Actinobacteria & identified in gut microbiota of IBD patients & $\mathrm{BO}$ \\
\hline 33 & g_ & s_ & 0.2 & Actinobacteria & Identified in fecal microbiota of pediatric IBD patients & $\mathrm{BP}$ \\
\hline 34 & Adlercreutzia & $s_{-}$ & 0.1 & Actinobacteria & Causes dysbiosis in IBD patients & $\mathrm{BQ}$ \\
\hline 35 & Atopobium & $s_{-}$ & 0.1 & Actinobacteria & altered intestinal microbiota in Crohn's disease & $\mathrm{BR}$ \\
\hline 36 & Slackia & s_ & 0.7 & Actinobacteria & Alters human gut microbiome in Multiple Sclerosis & BS \\
\hline 37 & Prevotella & $s_{-}$ & 0.1 & Bacteroidetes & A microbial signature of Crohn's disease & $\mathrm{BZ}$ \\
\hline 38 & $g_{-}$ & $s_{-}$ & 0.4 & Bacteroidetes & Identified in gut microbiome of IBD patients & CA \\
\hline 39 & g_ & s_ & 0.2 & Bacteroidetes & Characterized in intestinal biopsies in IBD patients & $\mathrm{CB}$ \\
\hline 40 & g_ & $s_{-}$ & 0.2 & Bacteroidetes & Human gut microbe in Obesity and IBD & $\mathrm{CC}$ \\
\hline 41 & Cloacibacterium & $s_{-}$ & 0.7 & Bacteroidetes & $\begin{array}{l}\text { Identified in the rectum of human colorectal } \\
\text { adenoma patients }\end{array}$ & CG \\
\hline 42 & SHD-231 & $\mathrm{s}$ & 0.1 & Chloroflexi & Identified in the fecal microbiome of Gout patients & $\mathrm{CH}$ \\
\hline 43 & $g_{-}$ & $s_{-}$ & 0.1 & Cyanobacteria & Identified in the gut microbiome of IBD patients & $\mathrm{Cl}$ \\
\hline
\end{tabular}


TABLE 4 | Continued

\section{SI. No. Bacteria genus}

Bacteria species
Proportion Bacteria phylum Function in IBD

\begin{tabular}{ll}
\hline 44 & Calothrix \\
45 & Bacillus \\
46 & g__ \\
47 & Gemella \\
48 & Abiotrophia \\
49 & Alloiococcus \\
50 & Enterococcus \\
51 & Lactobacillus \\
52 & Lactococcus \\
53 & g_ \\
54 & g_ \\
55 & Christensenella \\
56 & Clostridium \\
57 & Pseudoramibacter_ \\
& Eubacterium
\end{tabular}

$\begin{array}{ll}58 & 9 \\ 59 & \text { Blautia } \\ 60 & \text { Coprococcus } \\ 61 & \text { Dorea }\end{array}$

62 Lachnospira

$63 \quad 9$

64 g_

65 Peptostreptococcus

66 Anaerotruncus

67 Oscillospira

68 Ruminococcus

69 g_

70

71

72

73

74

$\begin{array}{lll}82 & 9 \_ & s_{-} \\ 83 & \text { g_ } & s_{-} \\ 84 & \text { Ochrobactrum } & s_{-}\end{array}$

$\begin{array}{lll}85 & \text { g_ } & s_{-} \\ 86 & \text { g_ } & s_{-}\end{array}$

\section{1}

0.1

0.5

0.5

0.1

0.1

0.1
3.4

\section{Firmicutes}

Firmicutes

Firmicutes

Firmicutes

Firmicutes

Firmicutes

Firmicutes

Firmicutes

Firmicutes

Firmicutes

Firmicutes

Firmicutes

Firmicutes

Firmicutes

Firmicutes

Firmicutes

Firmicutes

\section{3}

0.1

\section{6}

\section{Schwartzia}

Selenomonas

g_

Anaerococcus

Finegoldia

g_

Bulleidia

\section{Coprobacillus}

Fusobacterium

Leptotrichia

S-

Firmicutes

Firmicutes

Firmicutes

Firmicutes

Firmicutes

Firmicutes

Firmicutes

Firmicutes

Firmicutes

Firmicutes

Firmicutes

Firmicutes

Firmicutes

Firmicutes

Firmicutes

Firmicutes

Firmicutes

Fusobacteria

Fusobacteria

Gemmatimonadetes

Lentisphaerae

Proteobacteria

Cyanobacteria

Firmicutes
(\%)

Identified gut microbe in IBD patients

Increases cytokine levels in IBD

Causes microbiota dysbiosis in IBD

Microbiome in New-Onset Crohn's Disease

Causes fecal microbial dysbiosis in IBD

Identified gut microbe in IBD patients

Induces experimental IBD

Maintains remission of ulcerative colitis

Used in the treatment of Crohn's disease

Gut microbe in IBD

A microbial signature of Crohn's disease

Identified gut microbe

causes infection of the gut in IBD

Metabolizes Linoleic acid in the Gut

Commensal gut bacteria in IBD

Butyrate-producing bacterial species in Gut

Butyrate-producing bacterial species in Gut

Causes dysfunction of the intestinal microbiome in IBD

Gut bacteria in Crohn's disease patients

Gut microbe in IBD

A microbial signature of Crohn's disease

Causes gut microbiota dysbiosis in IBD

Tissue associated intestinal microflora

Gut microbe in IBD patients

Dominant in gut microbiome of IBD patients

Gut microbe underlying the onset of IBD

Gut microbe in IBD

Causes dysfunction of the intestinal microbiome in IBD

Causes fecal microbial dysbiosis in IBD

Causes dysbiosis in colorectal cancer

A microbial signature of Crohn's disease

Microbe in Inflammatory Pouch Complications

Intestinal microbe in colorectal cancer

Gut microbe in Gl diseases

Fecal-associated and mucosalassociated

microbiota in irritable bowel syndrome patients

Alters Gut Microbiota in Psoriatic Arthritis

Identified from colonic biopsies of IBD patients

Causes gut mucosal inflammation in Rheumatoid arthritis patients

Identified gut microbe in IBD patients

Normal gut microbe

Causes early bacterial dependent induction of inducible nitric oxide synthase (iNOS) in epithelial cells in experimental colitis

4.7 Proteobacteria

Intestinal microbe in children with severe and complicated acute viral gastroenteritis

Causes chronic inflammation in IBD
NCBI genome database link

CK

CK

$\mathrm{CL}$

$\mathrm{CO}$

$\mathrm{CP}$

CS

CT

CV

CW

CX

DA

DB

DC

DE

DF

DG

$\mathrm{DH}$

DI

DJ

DK

DP

DQ

DR

DT

DU

DV

DW

DX

DZ

EA

EB

EC

EE

EF

El

EJ

EL

EN

EO

EP

EQ

EU

EV

FB 
TABLE 4 | Continued

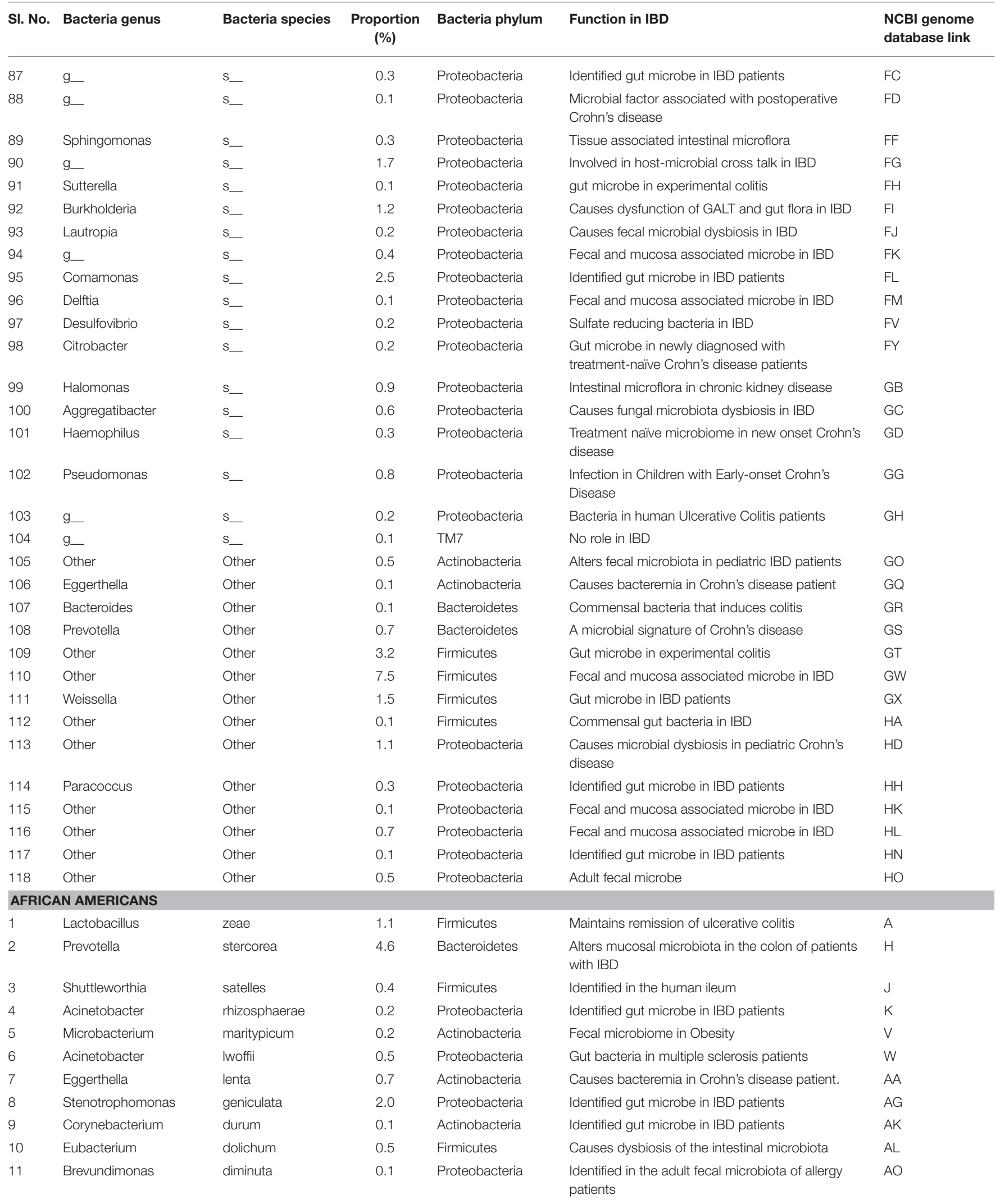


TABLE 4 | Continued

\section{SI. No. Bacteria genus}

Bacteria species

Proporti

(\%)

Lysinibacillus
Staphylococcus
Peptostreptococcus
Pseudomonas
Propionibacterium
Methanobrevibacter
g_
g_

boronitolerans

aureus

anaerobius

alcaligenes

acnes

s_

s_

s_

$$
\text { g_ }
$$

Varibaculum

Corynebacterium

\section{Arthrobacter}

Slackia

Chryseobacterium

Cloacibacterium

s-

$s_{-}$

s-

s-

s_

$s^{-}$$$
\text { g_ }
$$

$$
\text { Bacillus }
$$

Alloiococcus

Lactobacillus

Lactococcus

$$
\text { g_ }
$$

$$
\text { g_ }
$$

Christensenella

Clostridium

Pseudoramibacter_Eubacterium

$$
\text { Lachnospira }
$$

$$
\text { g_ }
$$

Peptostreptococcus

Oscillospira

Ruminococcus

$$
\text { g_ }
$$

\section{Acidaminococcus}

Phascolarctobacterium

Schwartzia

Selenomonas

Anaerococcus

Finegoldia

g_

Bulleidia

$51 \quad$ Fusobacterium

52 Leptotrichia

53 g_

54 Ochrobactrum

s-

s_

s_

$\begin{array}{cl}5.0 & \text { Firmicutes } \\ 0.2 & \text { Firmicutes } \\ 24.9 & \text { Firmicutes } \\ 1.1 & \text { Proteobacteria } \\ 0.6 & \text { Actinobacteria } \\ 0.4 & \text { Euryarchaeota } \\ 0.2 & \text { Acidobacteria } \\ 4.3 & \text { Acidobacteria }\end{array}$

0.3

0.6

0.5

0.2

0.2

0.3

0.2

0.2

0.2
2.7

2.7
6.0

0.2

0.3

0.1

1.3

0.2

0.4

0.9

0.3

0.3

0.4

0.2

2.2

0.4

0.4

0.1

0.5

0.5

0.4

0.8

Actinobacteria

Actinobacteria

Actinobacteria

Actinobacteria

Actinobacteria

Bacteroidetes

Cyanobacteria

Firmicutes

Firmicutes

Firmicutes

Firmicutes

Firmicutes

Firmicutes

Firmicutes

Firmicutes

Firmicutes

Firmicutes

Firmicutes

Firmicutes

Firmicutes

Firmicutes

Firmicutes

Firmicutes

Firmicutes

Firmicutes

Firmicutes

Firmicutes

Firmicutes

Firmicutes

Firmicutes

Fusobacteria

Fusobacteria

Proteobacteria
Function in IBD

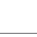

Identified gut microbe in IBD patients
Causes Crohn's disease
Causes dysbiosis in IBD
Identified in the gut microbiota of IBD
Intestinal microbe in Liver disease
Identified in the gut of IBD
Identified in human gut microbiota
Identified in the gut microbiome of Type 2 Diabetes
patients

Bacteroidetes

Gemmatimonadetes

Identified in gut microbiota in IBD

Identified in the gut of a premature infant

Causes experimental colitis

Fecal microflora in chronic IBD patients

Alters human gut microbiome in Multiple Sclerosis

Fecal and mucosa associated microbe in IBD

Identified in the rectum of human colorectal

adenoma patients

Identified in human gut microbiota

Increases cytokine levels in IBD

Identified gut microbe in IBD patients

Maintains remission of ulcerative colitis

Used in the treatment of Crohn's disease

Gut microbe in IBD

A microbial signature of Crohn's disease

Identified gut microbe

causes infection of the gut in IBD

Metabolizes Linoleic acid in the Gut

Gut bacteria in Crohn's disease patients

A microbial signature of Crohn's disease

Causes gut microbiota dysbiosis in IBD

Gut microbe in IBD patients

Dominant in gut microbiome of IBD patients

Gut microbe underlying the onset of IBD

Gut microbe in IBD

Causes dysfunction of the intestinal microbiome in IBD

Causes fecal microbial dysbiosis in IBD

Causes dysbiosis in colorectal cancer

Microbe in Inflammatory Pouch Complications

Intestinal microbe in colorectal cancer

Gut microbe in Gl diseases

Fecal-associated and mucosalassociated microbiota in irritable bowel syndrome patients Identified from colonic biopsies of IBD patients

NCBI genome database link

AT

$\mathrm{AU}$

AW

$\mathrm{AX}$

BA

$\mathrm{BB}$

$\mathrm{BC}$

$\mathrm{BD}$

BE

$\mathrm{BH}$

BI

$\mathrm{BL}$

BS

CF

CG

CJ

$\mathrm{CL}$

CT

CW

CX

DA

DB

DC

DE

DF

DK

DQ

DR

DU

DV

DW

DX

DZ

EA

EB

EE

EF

El

EJ

EN

Causes gut mucosal inflammation in Rheumatoid EO arthritis patients

Identified gut microbe in IBD patients EP

Causes early bacterial dependent induction of EU inducible nitric oxide synthase (iNOS) in epithelial cells in experimental colitis

Intestinal microbe in children with severe and EV complicated acute viral gastroenteritis 
TABLE 4 | Continued

\begin{tabular}{|c|c|c|c|c|c|c|}
\hline SI. No. & Bacteria genus & Bacteria species & $\begin{array}{l}\text { Proportion } \\
\text { (\%) }\end{array}$ & Bacteria phylum & Function in IBD & $\begin{array}{l}\text { NCBI genome } \\
\text { database link }\end{array}$ \\
\hline 56 & g_ & $s_{-}$ & 0.1 & Proteobacteria & Involved in host-microbial cross talk in IBD & $F G$ \\
\hline 57 & Sutterella & s_ & 0.2 & Proteobacteria & Gut microbe in experimental colitis & $\mathrm{FH}$ \\
\hline 58 & Burkholderia & $s_{-}$ & 0.4 & Proteobacteria & Causes dysfunction of GALT and gut flora in IBD & $\mathrm{Fl}$ \\
\hline 59 & Lautropia & s_ & 0.2 & Proteobacteria & Causes fecal microbial dysbiosis in IBD & FJ \\
\hline 60 & Comamonas & s_ & 0.4 & Proteobacteria & Identified gut microbe in IBD patients & $\mathrm{FL}$ \\
\hline 61 & Ralstonia & $s_{-}$ & 0.2 & Proteobacteria & $\begin{array}{l}\text { Microbiota in the Mucosa of Patients With Ulcerative } \\
\text { Colitis }\end{array}$ & FP \\
\hline 62 & Bilophila & s_ & 0.1 & Proteobacteria & Causes irritable bowel syndrome & FU \\
\hline 63 & Desulfovibrio & s_ & 0.1 & Proteobacteria & Sulfate reducing bacteria in IBD & FV \\
\hline 64 & Halomonas & s_ & 0.7 & Proteobacteria & Intestinal microflora in chronic kidney disease & GB \\
\hline 65 & g_ & s_ & 0.1 & Proteobacteria & Microbe in colon tissue from IBD subjects & GE \\
\hline 66 & Acinetobacter & s_ & 0.7 & Proteobacteria & Tissue associated intestinal microflora & GF \\
\hline 67 & Pseudomonas & $s_{-}$ & 0.1 & Proteobacteria & $\begin{array}{l}\text { Infection in Children with Early-onset Crohn's } \\
\text { Disease }\end{array}$ & GG \\
\hline 68 & g_ & $s_{-}$ & 0.8 & TM7 & No role in IBD & \\
\hline 69 & Other & Other & 0.3 & Actinobacteria & Alters fecal microbiota in pediatric IBD patients & $\mathrm{GO}$ \\
\hline 70 & Eggerthella & Other & 0.1 & Actinobacteria & Causes bacteremia in Crohn's disease patient & $\mathrm{GQ}$ \\
\hline 71 & Bacteroides & Other & 0.7 & Bacteroidetes & Commensal bacteria that induces colitis & GR \\
\hline 72 & Prevotella & Other & 3.2 & Bacteroidetes & A microbial signature of Crohn's disease & GS \\
\hline 73 & Other & Other & 2.8 & Firmicutes & Gut microbe in experimental colitis & GT \\
\hline 74 & Paenibacillus & Other & 0.1 & Firmicutes & Gut microbe in a healthy infant & GU \\
\hline 75 & Other & Other & 2.2 & Firmicutes & Fecal and mucosa associated microbe in IBD & GW \\
\hline 76 & Paracoccus & Other & 0.1 & Proteobacteria & Identified gut microbe in IBD patients & $\mathrm{HH}$ \\
\hline 77 & Other & Other & 0.6 & Proteobacteria & Fecal and mucosa associated microbe in IBD & $\mathrm{HL}$ \\
\hline 78 & Other & Other & 0.1 & Proteobacteria & Adult fecal microbe & $\mathrm{HO}$ \\
\hline 79 & Other & Other & 0.4 & Proteobacteria & Mucosal and fecal microbe & $\mathrm{HP}$ \\
\hline 80 & Acinetobacter & Other & 2.2 & Proteobacteria & $\begin{array}{l}\text { Tissue associated intestinal microflora in colitis } \\
\text { patients }\end{array}$ & $\mathrm{HQ}$ \\
\hline 81 & Pseudomonas & Other & 0.2 & Proteobacteria & $\begin{array}{l}\text { Gut microbe in children with early onset Crohn's } \\
\text { disease }\end{array}$ & $\mathrm{HR}$ \\
\hline
\end{tabular}

Specific Information of functions was adapted from NCBI Genome Database (https://www.ncbi.nlm.nih.gov/genome/).

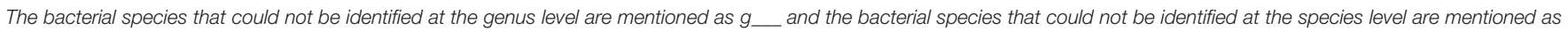
s..

compared to adjacent healthy colon specimens. We suggest that pathogenic bacteria belonging to these two phyla have a greater impact on colon motility function in colitis patients (Tables 1, 3). Although the incidence of IBD is increasing among African Americans (AA), the underlying causes are completely unknown (Sofia et al., 2014). Our study further highlight a significant disparity in bacterial dysbiosis among AA compared to CA colitis patients (Figure 3 ).

CA specimens had significantly higher levels of Fusobacterium, Parabacteroides, Citrobacter, Haemophilus, Acinetobacter, Pseudomonas, and Stenotrophomonas. Fusobacterium nucleatum is known to have a well-characterized role in the oral cavity. We have determined that Fusobacterium can be recovered from human full thickness colon specimens and this could indicate their ability to survive and proliferate inside host cells. Parabacteroides was found to be dominant in the acute phase of IBD in CA patients. Citrobacter is an epithelial cell adherent pathogen and can subvert inflammation in colitis.
Pseudomonas interacts with the mucosal layer of colon and disrupts the mucosal barrier integrity leading to colitis in CA patients.

The AA specimens had significantly higher levels of Prevotella and Clostridia. Prevotella augments T-helper cells mediated colon mucosal inflammation by activating Toll-like receptor 2 leading to production of T-helper cells polarizing cytokines by antigen-presenting cells, including interleukins. In addition, Prevotella induce epithelial cells to produce interleukins and cytokines that can promote recruitment of neutrophils and mucosal T-helper cell immune responses. Prevotella can mediate inflammation of the mucosa leading to the circulation of bacteria, bacterial products and other inflammatory mediators. Prevotella could augment release of inflammatory mediators from immune cells and various stromal cells in colitis in AA patients. Clostridium can disrupt gut immune dormancy and cause infectious colitis in AA patients. Collectively, our data suggest that the presence of pathogenic bacteria in AA 
TABLE 5 | Proportions of bacterial species of unknown function colonized in full thickness colon of colitis patients.

\begin{tabular}{|c|c|c|c|c|c|c|}
\hline SI. No. & Bacteria genus & Bacteria species & Proportion (\%) & Bacteria phylum & Function in IBD & NCBI genome database link \\
\hline \multicolumn{7}{|c|}{ ADJACENT HEALTHY COLON } \\
\hline 1 & Micrococcus & luteus & 0.01 & Actinobacteria & No role in IBD & $x$ \\
\hline 2 & Arthrobacter & $\mathrm{s}$ & 0.01 & Actinobacteria & No role in IBD & $\mathrm{BL}$ \\
\hline 3 & Propionicimonas & S_ & 0.01 & Actinobacteria & No role in IBD & $\mathrm{BN}$ \\
\hline 4 & Paludibacter & $s_{-}$ & 0.01 & Bacteroidetes & No role in IBD & BW \\
\hline 5 & Chryseobacterium & s_ & 0.02 & Bacteroidetes & No role in IBD & CF \\
\hline 6 & Calothrix & $\mathrm{s}$ & 0.03 & Cyanobacteria & No role in IBD & CK \\
\hline 7 & Novosphingobium & $\mathrm{s}$ & 0.02 & Proteobacteria & No role in IBD & FE \\
\hline \multicolumn{7}{|c|}{ DISEASED COLON (ULCERATIVE COLITIS) } \\
\hline 1 & Micrococcus & luteus & 0.01 & Actinobacteria & No role in IBD & $x$ \\
\hline 2 & Arthrobacter & $s_{-}$ & 0.02 & Actinobacteria & No role in IBD & $\mathrm{BL}$ \\
\hline 3 & Propionicimonas & $s_{-}$ & 0.1 & Actinobacteria & No role in IBD & $\mathrm{BN}$ \\
\hline 4 & Paludibacter & s_ & 0.03 & Bacteroidetes & No role in IBD & BW \\
\hline 5 & Chryseobacterium & s_ & 0.1 & Bacteroidetes & No role in IBD & $\mathrm{CF}$ \\
\hline 6 & Calothrix & $s_{-}$ & 0.1 & Cyanobacteria & No role in IBD & $\mathrm{CK}$ \\
\hline 7 & Novosphingobium & s_ & 0.04 & Proteobacteria & No role in IBD & FE \\
\hline \multicolumn{7}{|c|}{ DISEASED COLON (CROHN'S COLITIS) } \\
\hline 1 & Micrococcus & luteus & 0.02 & Actinobacteria & No role in IBD & $x$ \\
\hline 2 & Arthrobacter & s_ & 0.2 & Actinobacteria & No role in IBD & $\mathrm{BL}$ \\
\hline 3 & Propionicimonas & s_ & 0.02 & Actinobacteria & No role in IBD & $\mathrm{BN}$ \\
\hline 4 & Paludibacter & $s_{-}$ & 0.02 & Bacteroidetes & No role in IBD & BW \\
\hline 5 & Chryseobacterium & s_ & 0.3 & Bacteroidetes & No role in IBD & CF \\
\hline 6 & Calothrix & $\mathrm{s}$ & 0.1 & Cyanobacteria & No role in IBD & CK \\
\hline 7 & Novosphingobium & $s_{-}$ & 0.01 & Proteobacteria & No role in IBD & FE \\
\hline
\end{tabular}

Specific Information of functions was adapted from NCBI Genome Database (https://www.ncbi.nlm.nih.gov/genome/).

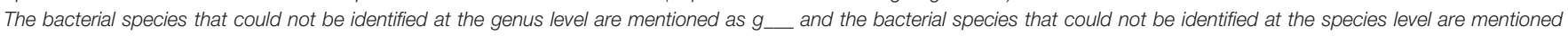
as s_.

full thickness diseased specimens could adversely affect colon motility.

Additionally, our data in UC and CC specimens show the presence of several orange (Prevotella, Peptostreptococcus, Eubacterium, Fusobacterium, and Campylobacter), red (Porphyromonas), purple (Veillonella), and yellow (Streptococcus) complex putative oral pathogens known to cause gingivitis and periodontitis among IBD patients (Tables 1, 3). Previous studies using mucosal biopsies and feces have shown that gut microbiota in bowel diseases is characterized by an increase in certain phyla such as Proteobacteria, Firmicutes, genus Bifidobacterium, as well as a reduction in the amounts of genera Ruminococcus, Clostridia and (in some cases) Faecalibacterium (Lane et al., 2017; Nishida et al., 2018). However, none of the earlier studies using feces have shown a shift in the balance between Phyla Bacteroidetes and Firmicutes among UC or CC patients; even though this was observed in healthy individuals (Mariat et al., 2009; Koliada et al., 2017). In contrary to our results, one study using mucosal biopsies has shown a significantly decreased Firmicutes to Bacteroidetes ratio in both UC and CC compared with controls (Kabeerdoss et al., 2015). Collectively, our data suggest that the putative oral pathogens found in diseased colon specimens may modulate the proportion of non-detrimental gut bacteria, thus potentially worsening the condition of the colon in colitis patients.

Oral bacterial species like Porphyromonas, Peptostreptococcus, Eubacterium, Fusobacterium, Streptococcus salivarius, S. mitis, S. bovis, Veillonella spp., Staphylococcus aureus, S. epidermidis, and Campylobacter spp. can convert nitrate to nitrite. A large amount of bioactive NO is found in the gastrointestinal tract, generated by dietary sources and by conversion of anaerobic bacteria in the oral cavity, or by anaerobic reaction with nitrate in the colon by Escherichia coli spp. The entero-salivary nitrate conversion pathway provides a rich source of bioactive NO and nitrate-reducing bacteria, such as Veillonella. In this pathway, nitrate is obtained by the salivary gland and is then concentrated in the saliva. Various facultative anaerobic bacteria on the top of the tongue effectively reduces nitrate to nitrite. The bacteria then use the nitrate and the nitrite as electron acceptors in their respiration process. This also helps the host in the first steps of converting nitrate to NO. The salivary nitrate then reaches the systemic circulation, various enzymatic reactions occur leading to reduction to $\mathrm{NO}$, and other reactive nitrogen intermediates. The oral cavity plays an important role the production of nitric oxide, and specifically, employs the nitrate-nitrite-NO pathway in the oral cavity. It is well known that oral cavity bacteria can migrate to the colon. Taken together, our data suggest that the 


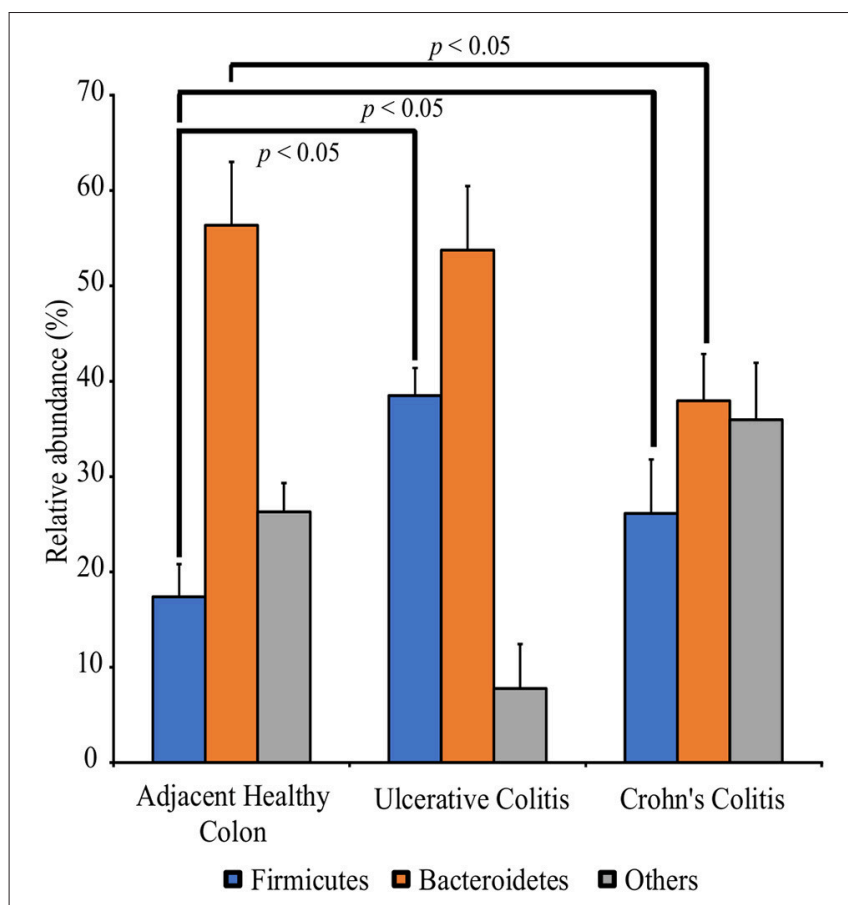

FIGURE 2 | Perturbation of the full thickness colon microbiome in Colitis specimens as compared to adjacent healthy specimens. Specimens are categorized into adjacent healthy colon $(n=13)$, Ulcerative colitis $(\cup C, n=13)$ and Crohn's colitis (CC, $n=13$ ). Data represented are the mean of relative abundances of each phylum identified in specimens belonging to each group while error bars indicate standard error. Diseased specimens demonstrate a balance between the Phyla Firmicutes and Bacteroidetes. Conversely, healthy colon specimens demonstrate a significantly higher proportion of Phyla Bacteroidetes. ${ }^{\star} p<0.05$ by Mann-Whitney $\cup$ Test ( $n=13$ under each group).

putative oral pathogens found in diseased colon specimens may survive by exploiting the nitrate-nitrite-NO pathway to modulate the proportion of non-detrimental gut bacteria, thus potentially worsening the condition of colon in colitis patients (Figure 4).

Previous studies suggest that enteric neurons and smooth muscle mediated gut motility is impaired in colitis patients (Snape et al., 1991; Vermillion et al., 1993). IBD associated gut inflammation affects the morphological and functional changes in the myenteric/enteric nervous system (ENS) and nitric oxide (NO) synthesis (Takahashi, 2003; Kono et al., 2004). Experimental studies have also shown that gut bacteria have a role in oxidative stress induced gut inflammation by controlling metabolic endotoxemia in obese mice (Cani et al., 2008). We have shown that polybacterial oral infection decrease the expression of nNOS and NRF2-phase II enzymes in the gut and this could lead to impaired colon motility (Gangula et al., 2015; Walker et al., 2018).

Some of the gut bacteria we have identified in the full thickness colon specimens in the present study, including Bacteroides, Prevotella, Pseudomonas, etc., have been identified in colon mucosal biopsies in earlier studies (Bibiloni et al., 2006). These bacteria evoke inflammatory responses affecting the innermost lining of colon. Many specific beneficial bacteria, including members of Bacteroides and Prevotella groups, C. coccoides, and
Lactic acid bacteria were known to be decreased in colitis patients (Gibson et al., 1991). Specimens used in prior studies were colon mucosal biopsies or stool samples; but not full thickness colon specimens (Gibson et al., 1991; Bibiloni et al., 2006). Fullthickness colon consists of four layers of tissue including mucosa, submucosa, muscularis, and serosa.

Novel to this research design, full thickness colon specimens were obtained because colitis patients often experience colon motility abnormalities (Snape et al., 1991; Annese et al., 1997; Vrees et al., 2002). Several lines of evidence suggest that nitrergic neurons that releases $\mathrm{NO}$ via nNOS are known to play a pivotal role in colon motility (Kono et al., 2004; Winston et al., 2013). Previous studies have demonstrated that nitrergic neurons are degenerated in colitis (Onori et al., 2005; Sung et al., 2006). Recent studies from our laboratory indicate that nNOS, as well as antioxidants (NRF2 regulated-Phase II enzymes) protein expression are down-regulated in diseased colon specimens (Myers et al., 2014; Gangula et al., 2017). Furthermore, our previous studies demonstrated that polybacterial infection led to a decrease in nNOS, NRF2 and antioxidants protein expression in the colon tissues (Gangula et al., 2015). In addition, studies have shown that NO may play homeostatic role in gut inflammation (Kolios et al., 2004). Taken together, our data suggest that elevated levels of oral and gut pathogens in diseased colon full thickness specimens could contribute to impaired nNOS-NO-NRF2-Phase II system and colon motility abnormalities in IBD patients (Figure 4).

To our knowledge, our study is the first to report the presence of several microbiota of unknown function in IBD including Micrococcus luteus, Chloracidobacteria, Arthrobacter, Propionicimonas, Paludibacter, Chryseobacterium, Calothrix, and Novosphingobium (Table 5). These new microbiota members have not been identified in mucosal/fecal specimens in previous studies, suggesting that these bacteria are primarily colonized in the neuromuscular compartment. Additional studies are warranted to characterize the novel bacteria and investigate their specific role in colon motility and constipation in IBD patients.

In summary, this study have identified specific bacterial pathogens potentially associated with colon motility in IBD patients. The observations showed that some putative oral pathogens belonging to the Phyla Firmicutes (Streptococcus, Staphylococcus, Peptostreptococcus), and Fusobacteria (Fusobacterium) dominated in the microbiomes of CC and UC diseased specimens and might involve the modulation of colon motility in IBD.

\section{STUDY LIMITATIONS}

The limitations of the study include the smaller sample size across disease and race groups making this as a preliminary study. In spite of the limitations in sample size and the fact that some of the identified bacteria were not significantly altered in colitis specimens, we were still able to observe differences in the microbiome between CA and AA colitis patients. This could be due to amplicon sequencing of a shorter conserved region of 16S rRNA gene instead of in depth shotgun sequencing. 


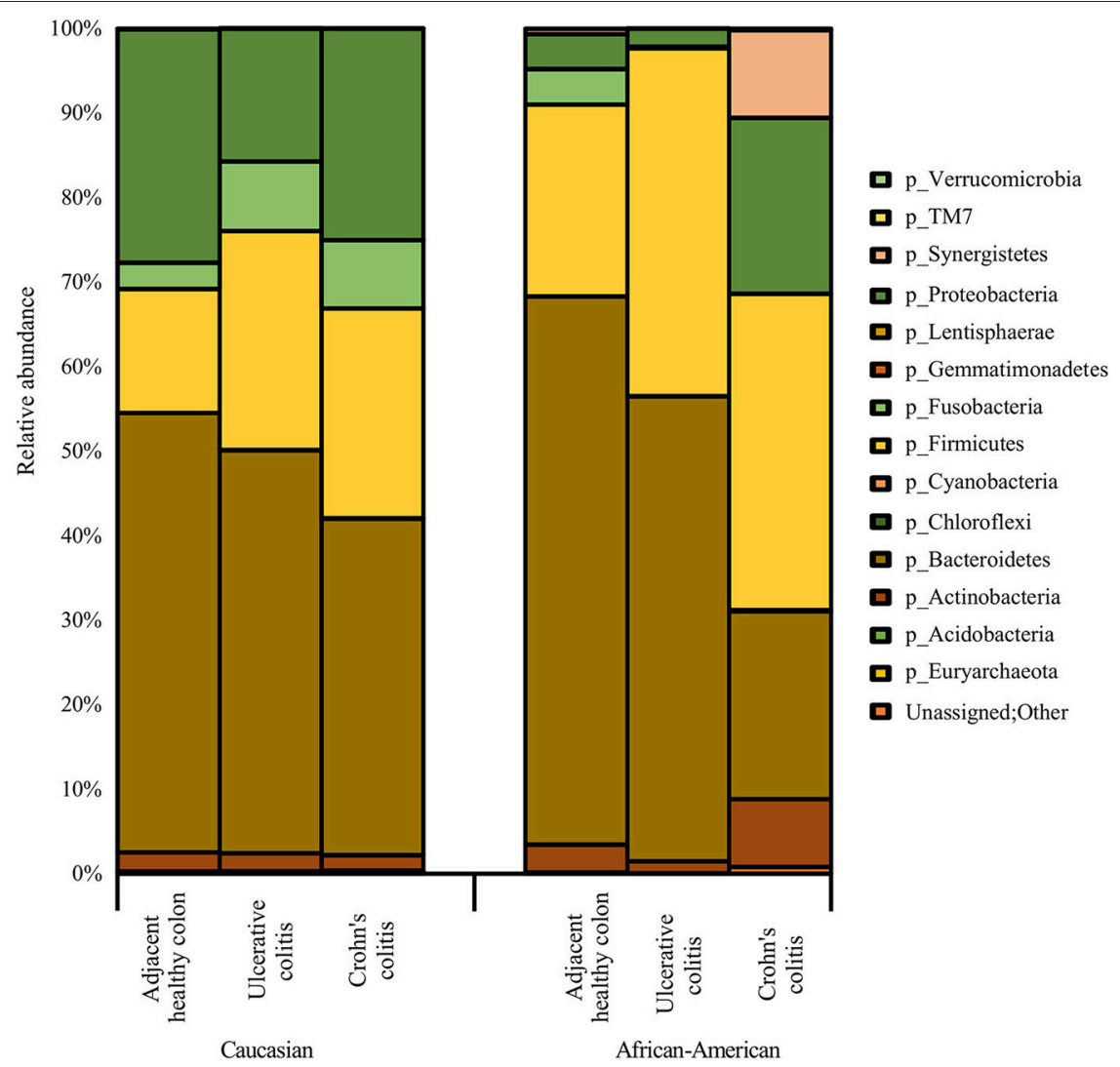

FIGURE 3 | Summary of major bacterial taxa showing the relative abundance of oral and gut bacteria at the Phylum level in the colitis and adjacent healthy specimen groups under each race. Data represented are the mean of relative abundances of each Phyla detected in samples belonging to each group. The dominant phyla across all samples (both diseased and healthy specimens) were Bacteroidetes, followed by Firmicutes and Proteobacteria. Other major phyla observed among these specimens also include Actinobacteria, Fusobacteria, and Synergistetes. The Phylum Proteobacteria did not show any significant difference between healthy colon specimens and diseased colon specimens. A larger proportion of unassigned bacteria $(0.3 \%)$ was identified in AA Crohn's Colitis patients compared to other groups.
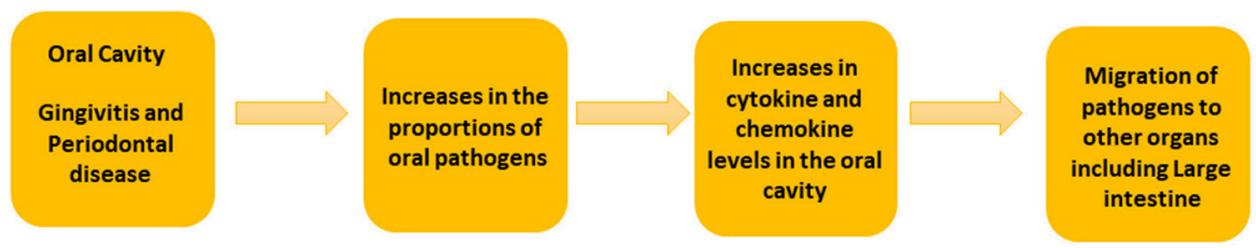

\section{Increase in} proinflammatory cytokines and chemokines in

large intestine
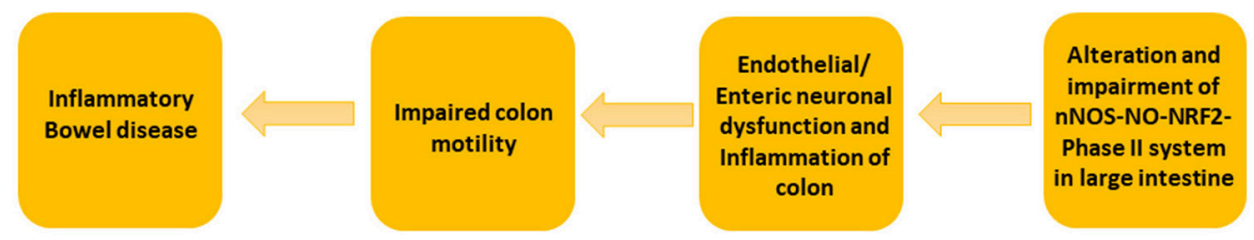

FIGURE 4 | Schematic Representation of the suggested mechanism involved in the development of colitis by oral and gut microbiome. We propose that the increase in the concentrations of putative oral pathogens elevates the cytokine and chemokine levels in oral cavity. When putative oral pathogens travel to the gut, they can colonize locally and lead to the elevated levels of proinflammatory cytokines. This can effect on nNOS-NO-NRF2-Phase II system in the large intestine and could lead to colon dysmotility and colitis. 
Moreover, we did not profile the oral microbiome from oral specimens (dental plaque, etc.) in the same IBD patients from whom full thickness colon specimens were collected. Finally, host-microbiome interaction studies are needed to better discern specific roles of the oral and gut bacteria in the development of colitis. Future studies are aimed to collect oral and fecal specimens therefore a comparative experiments in regards to changes in microbiome, along with specific key proteins will be conducted from the same patient.

\section{AUTHOR CONTRIBUTIONS}

VD, SM, KS, SP, SS, PG, and MT have contributed both for data analysis and manuscript preparation. DS, CF-D, LK, SA, and JS have contributed in manuscript preparation.

\section{REFERENCES}

Agossa, K., Dendooven, A., Dubuquoy, L., Gower-Rousseau, C., DelcourtDebruyne, E., and Capron, M. (2017). Periodontal manifestations of inflammatory bowel disease: emerging epidemiologic and biologic evidence. J. Periodontal Res. 52, 313-324. doi: 10.1111/jre.12422

Annese, V., Bassotti, G., Napolitano, G., Usai, P., Andriulli, A., and Vantrappen, G. (1997). Gastrointestinal motility disorders in patients with inactive Crohn's disease. Scand. J. Gastroenterol. 32, 1107-1117.

Atarashi, K., Suda, W., Luo, C., Kawaguchi, T., Motoo, I., Narushima, S., et al. (2017). Ectopic colonization of oral bacteria in the intestine drives TH1 cell induction and inflammation. Science 358, 359-365. doi: 10.1126/science.aan4526

Bassotti, G., Antonelli, E., Villanacci, V., Salemme, M., Coppola, M., and Annese, V. (2014). Gastrointestinal motility disorders in inflammatory bowel diseases. World J. Gastroenterol. 20, 37-44. doi: 10.3748/wjg.v20.i1.37

Bibiloni, R., Mangold, M., Madsen, K. L., Fedorak, R. N., and Tannock, G. W. (2006). The bacteriology of biopsies differs between newly diagnosed, untreated, Crohn's disease and ulcerative colitis patients. J. Med. Microbiol. 55, 1141-1149. doi: 10.1099/jmm.0.46498-0

Cani, P. D., Bibiloni, R., Knauf, C., Waget, A., Neyrinck, A. M., Delzenne, N. M., et al. (2008). Changes in gut microbiota control metabolic endotoxemiainduced inflammation in high-fat diet-induced obesity and diabetes in mice. Diabetes 57, 1470-1481. doi: 10.2337/db07-1403

Caporaso, J. G., Bittinger, K., Bushman, F. D., DeSantis, T. Z., Andersen, G. L., and Knight, R. (2010a). PyNAST: a flexible tool for aligning sequences to a template alignment. Bioinform. 26, 266-267. doi: 10.1093/bioinformatics/btp636

Caporaso, J. G., Kuczynski, J., Stombaugh, J., Bittinger, K., Bushman, F. D., Costello, E. K., et al. (2010b). QIIME allows analysis of highthroughput community sequencing data. Nat. Methods 7, 335-336. doi: $10.1038 /$ nmeth.f.303

Edgar, R. C. (2010). Search and clustering orders of magnitude faster than BLAST. Bioinformatics 26, 2460-2461. doi: 10.1093/bioinformatics/btq461

Gangula, P., Ravella, K., Chukkapalli, S., Rivera, M., Srinivasan, S., Hale, A., et al. (2015). Polybacterial periodontal pathogens alter vascular and gut $\mathrm{BH}_{4} / \mathrm{nNOS} / \mathrm{NRF} 2-$ phase II enzyme expression. PLOS ONE 10:e129885. doi: 10.1371/journal.pone.0129885.

Gangula, P. R., Smoot, D. T., Izban, M., Ballard, B., and Adunyah, S. (2017). "Expression of NO and NRF2 enzymes in Human Colitis," in Proceedings of the RCMI Translational Science 2017 Conference (Washington, DC), 78.

Geboes, K., and Collins, S. (1998). Structural abnormalities of the nervous system in Crohn's disease and ulcerative colitis. Neurogastroenerol. Motil. 10, 189-202.

Gibson, G. R., Cummings, J. H., and Macfarlane, G. T. (1991). Growth and activities of sulphate-reducing bacteria in gut contents of healthy subjects and patients with ulcerative colitis. FEMS Microbiol. Lett. 86, 103-111. doi: 10.1111/j.1574-6968.1991.tb04799.x

\section{ACKNOWLEDGMENTS}

The authors sincerely thank University of North Carolina at Chapel Hill School of Medicine- Microbiome Core Facility (UNC; MC) for performing amplicon sequencing. We thank $\mathrm{CHTN}$ for providing diseased and healthy specimens. We further thank dental student doctors Mr. A. Arab and J. Hodges for their initial contribution on this project. The Meharry Bioinformatics Core is funded in part by NIH grants MD007593 and MD007586. SA is supported by NIMHD R-CTR grant No. 54544MD007593 and NCI grant No. 5454CA163069. This work is supported in part by 1SC1GM121282 funded to PG. In addition, Research reported in this publication was supported by the National Institute of General Medical Sciences of the National Institutes of Health under Award Number SG1GM121282 (PG).

Gower, J. C. (2005). "Principal coordinates analysis," in Encyclopedia of Biostatistics, eds P. Armitage and T. Colton, (John Wiley \& Sons Ltd). doi: 10.1002/0470011815

Haas, B. J., Gevers, D., Earl, A. M., Feldgarden, M., Ward, D. V., Giannoukos, G., et al. (2011). Chimeric $16 \mathrm{~S}$ rRNA sequence formation and detection in Sanger and 454-pyrosequenced PCR amplicons. Genome Res. 21, 494-504. doi: $10.1101 /$ gr.112730.110

Hajishengallis, G. (2015). Periodontitis: from microbial immune subversion to systemic inflammation. Nat. Rev. Immunol. 15, 30-44. doi: 10.1038/ nri3785

Kabeerdoss, J., Jayakanthan, P., Pugazhendhi, S., and Ramakrishna, B. S. (2015). Alterations of mucosal microbiota in the colon of patients with inflammatory bowel disease revealed by real time polymerase chain reaction amplification of $16 \mathrm{~S}$ ribosomal ribonucleic acid. Indian J Med Res. 142, 23-32. doi: 10.4103/0971-5916.162091

Keegan, K. P., Glass, E. M., and Meyer, F. (2016). MG-RAST, a metagenomics service for analysis of microbial community structure and function. Methods Mol. Biol. 1399, 207-233. doi: 10.1007/978-1-4939-3369-3_13

Koliada, A., Syzenko, G., Moseiko, V., Budovska, L., Puchkov, K., Perederiy, V., et al. (2017). Association between body mass index and Firmicutes/Bacteroidetes ratio in an adult Ukrainian population. BMC Microbiol. 17:120. doi: 10.1186/s12866-017-1027-1

Kolios, G., Valatas, V., and Ward, S. G. (2004). Nitric oxide in inflammatory bowel disease: a universal messenger in an unsolved puzzle. Immunology 113, 427-437. doi: 10.1111/j.1365-2567.2004.01984.x

Kono, T., Chisato, N., Ebisawa, Y., Asama, T., Sugawara, M., Ayabe, T., et al. (2004). Impaired nitric oxide production of the myenteric plexus in colitis detected by a new bioimaging system. J. Surg. Res. 117, 329-336. doi: 10.1016/j.jss.2003.11.004

Lane, E. R., Zisman, T. L., and Suskind, D. L. (2017). The microbiota in inflammatory bowel disease: current and therapeutic insights. J. Inflamm. Res. 10, 63-73. doi: $10.2147 /$ JIR.S116088

Lira-Junior, R., and Figueredo, C. M. (2016). Periodontal and inflammatory bowel diseases: is there evidence of complex pathogenic interactions? World J. Gastroenterol. 22, 7963-7972. doi: 10.3748/wjg.v22.i35.7963

Mariat, D., Firmesse, O., Levenez, F., Guimaraes, V. D., Sokol, H., Dore, J., et al. (2009). The Firmicutes/Bacteroidetes ratio of the human microbiota changes with age. BMC Microbiol. 9:123. doi: 10.1186/1471-2180-9-123

Myers, J. N., Schaffer, M. W., Korolkova, O. Y., Williams, A. D., Gangula, P. R., and M'Koma, A. E. (2014). Implications of the colonic deposition of free hemoglobin- $\alpha$ chain: a previously unknown tissue by-product in inflammatory bowel disease. Inflamm. Bowel Dis. 20, 1530-1547. doi: 10.1097/MIB.0000000000000144

Nishida, A., Inoue, R., Inatomi, O., Bamba, S., Naito, Y., and Andoh, A. (2018). Gut microbiota in the pathogenesis of inflammatory bowel disease. Clin. J. Gastroenterol. 11, 1-10. doi: 10.1007/s12328-017-0813-5 
Onori, L., Aggio, A., D’Alo, S., Muzi, P., Cifone, M. G., Mellillo, G., et al. (2005). Role of nitric oxide in the impairment of circular muscle contractility of distended, uninflamed mid-colon in TNBS-induced acute distal colitis in rats. World J. Gastroenterol. 11, 5677-5684. doi: 10.3748/wjg.v11.i36.5677

Poli, E., Lazzeretti, M., Grandi, D., Pozzoli, C., and Coruzzi, G. (2001). Morphological and functional alterations of the myenteric plexus in rats with TNBS-induced colitis. Neurochem. Res. 26, 1085-1093. doi: 10.1023/A:1012313424144

Popova, C., Dosseva-Panova, V., and Panov, V. (2013). Microbiology of periodontal diseases. a review. Biotechnol. Biotechnol. Equip. 27, 3754-3759. doi: 10.5504/BBEQ.2013.0027

Price, M. N., Dehal, P. S., and Arkin, A. P. (2010). FastTree 2-approximately maximum-likelihood trees for large alignments. PLoS ONE 5:e9490. doi: 10.1371/journal.pone.0009490.

Schloss, P. D., Westcott, S. L., Ryabin, T., Hall, J. R., Hartmann, M., Hollister, E. B., et al. (2009). Introducing mothur: open-source, platformindependent, community-supported software for describing and comparing microbial communities. Appl. Environ. Microbiol. 75, 7537-7541. doi: 10.1128/AEM.01541-09

Snape, W. J. Jr., Williams, R., and Hyman, P. E. (1991). Defect in colonic smooth muscle contraction in patients with ulcerative colitis. Am. J. Physiol. 261, G987-G991. doi: 10.1152/ajpgi.1991.261.6.G987

Sofia, M. A., Rubin, D. T., Hou, N., and Pekow, J. (2014). Clinical presentation and disease course of inflammatory bowel disease differs by race in a large tertiary care hospital. Dig Dis Sci. 59, 2228-2235. doi: 10.1007/s10620-014-3160-0

Strauss, J., Kaplan, G. G., Beck, P. L., Rioux, K., Panaccione, R., Devinney, R., et al. (2011). Invasive potential of gut mucosa-derived Fusobacterium nucleatum positively correlates with IBD status of the host. Inflamm. Bowel Dis. 17, 1971-1978. doi: 10.1002/ibd.21606

Sung, T. S., La, J. H., Kim, T. W., and Yang, I. S. (2006). Alteration of nitrergic neuromuscular transmission as a result of acute experimental colitis in rat. $J$. Vet. Sci. 7, 143-150. doi: 10.4142/jvs.2006.7.2.143

Takahashi, T. (2003). Pathophysiological significance of neuronal nitric oxide synthase in the gastrointestinal tract. J. Gastroenterol. 38, 421-430. doi: $10.1007 /$ s00535-003-1094-y
Vavricka, S. R., Manser, C. N., Hediger, S., Vogelin, M., Scharl, M., Biedermann, L., et al. (2013). Periodontitis and gingivitis in inflammatory bowel disease: a case-control study. Inflamm. Bowel. Dis. 19, 2768-2777. doi: 10.1097/01.MIB.0000438356.84263.3b

Vermillion, D. L., Huizinga, J. D., Riddell, R. H., and Collins, S. M. (1993). Altered small intestinal smooth muscle function in Crohn's disease. Gastroenterol. 104, 1692-1699.

Vrees, M. D., Pricolo, V. E., Ptenti, F. M., and Cao, W. (2002). Abnormal motility in patients with ulcerative colitis: the role of inflammatory cytokines. Arch. Surg. 137, 439-445. doi: 10.1001/archsurg.137.4.439

Walker, M. Y., Pratap, S., Southerland, J. H., Farmer-Dixon, C. M., Lakshmyya, K., and Gangula, P. R. (2018). Role of oral and gut microbiome in nitric oxide-mediated colon motility. Nitric Oxide 73, 81-88. doi: 10.1016/j.niox.2017.06.003

Winston, J. H., Li, Q., and Sarna, S. K. (2013). Paradoxical regulation of ChAT and nNOS expression in animal models of Crohn's colitis and ulcerative colitis. Am. J. Physiol. Gastrointest. Liver Physiol. 305, G295-302. doi: 10.1152/ajpgi.00052.2013

Zhao, L., Huang, Y., Lu, L., Yang, W., Huang, T., Lin, Z., et al. (2018). Saturated long-chain fatty acid-producing bacteria contribute to enhanced colonic motility in rats. Microbiome 6:107. doi: 10.1186/s40168-0180492-6

Conflict of Interest Statement: The authors declare that the research was conducted in the absence of any commercial or financial relationships that could be construed as a potential conflict of interest.

Copyright (c) 2019 Dinakaran, Mandape, Shuba, Pratap, Sakhare, Tabatabai, Smoot, Farmer-Dixon, Kesavalu, Adunyah, Southerland and Gangula. This is an openaccess article distributed under the terms of the Creative Commons Attribution License (CC BY). The use, distribution or reproduction in other forums is permitted, provided the original author(s) and the copyright owner(s) are credited and that the original publication in this journal is cited, in accordance with accepted academic practice. No use, distribution or reproduction is permitted which does not comply with these terms. 